ESAIM: PROCEEDINGS AND SURVEYS, December 2015, Vol. 52, p. 105-126

S. Dellacherie, F. Dubois, S. Fauve, R. Gatignol, Editors

\title{
PRELIMINARY RESULTS FOR THE STUDY OF THE GODUNOV SCHEME APPLIED TO THE LINEAR WAVE EQUATION WITH POROSITY AT LOW MACH NUMBER
}

\author{
Stéphane Dellacherie ${ }^{1}$, Jonathan Jung ${ }^{2}$ and Pascal Omnes ${ }^{3}$
}

\begin{abstract}
We introduce continuous tools to study the low Mach number behavior of the Godunov scheme applied to the linear wave equation with porosity on cartesian meshes. More precisely, we extend the Hodge decomposition to a weighted $L^{2}$ space in the continuous case and we study the properties of the modified equation associated to this Godunov scheme. This allows to partly explain the inaccuracy of the Godunov scheme at low Mach number on cartesian meshes and to propose two corrections: a first one named low Mach and a second one named all Mach. These results are preliminary since it remains to prove them in the discrete case.

Résumé. Nous introduisons des outils au niveau continu pour étudier le comportement du schéma de Godunov à bas nombre de Mach appliqué à l'équation des ondes avec porosité sur maillage cartésien. Plus précisément, nous étendons la décomposition de Hodge à un espace $L^{2}$ avec poids et nous étudions les propriétés de l'équation modifiée associée au schéma de Godunov. Cela permet de partiellement expliquer la perte de précision du schéma de Godunov à bas nombre de Mach sur maillage cartésien et de proposer deux corrections: une première dite bas Mach et une seconde dite tout Mach. Ces résultats sont préliminaires puisqu'il reste à les étendre au niveau discret.
\end{abstract}

\section{INTRODUCTION}

In a nuclear reactor core, the flow has a low Mach number, which means that the flow speed is small with respect to the sound speed. Then, it seems natural to use a low Mach model - that is incompressible when we neglect the heat sources - and related numerical methods to compute this kind of flow $[1,3]$. However, in some accidental cases, shock waves may appear in a nuclear reactor core, and thus one may wish to develop a numerical method that could also treat these accidental cases. Since a low Mach (or incompressible) model does not allow to consider shock waves, we consider a compressible model: the barotropic Euler equations. Finite volume Godunov type schemes applied to the compressible Euler system allow to compute the entropic solution of the compressible system [9,10] but are known to be inaccurate at low Mach number [12-14]. Indeed, these schemes are too diffusive at low Mach number and they diffuse the numerical solution to a wrong stationary

1 CEA, DEN, DM2S, STMF F-91191, Gif-sur-Yvette, France and Université Pierre et Marie Curie, LRC Manon and LJLL, 4 place Jussieu, 75252 Paris, cedex 05, France, stephane.dellacherie@cea.fr

2 EFREI \& AlliansTIC, 30-32 avenue de la République, 94800 Villejuif, France and Université Pierre et Marie Curie, LRC Manon and LJLL, 4 place Jussieu, 75252 Paris, cedex 05, France and LMA-IPRA, Université de Pau et des Pays de l'Adour, UMR CNRS 5142, Avenue de l'Université, 64013 Pau, France and INRIA Bordeaux Sud Ouest, Cagire Team, 351 Cours de la Libération, 33405 Talence, France, jonathan.jung@groupe-efrei.fr

${ }^{3}$ CEA, DEN, DM2S, STMF F-91191, Gif-sur-Yvette, France and Université Paris 13, Sorbonne Paris Cité, LAGA, CNRS UMR 7539, 99, Avenue J.-B. Clément F-93430 Villetaneuse Cedex, France, pascal.omnes@cea.fr

(C) EDP Sciences, SMAI 2015 
space [2] (except on triangular or tetrahedral meshes [7,11,16,18]). A low Mach correction is proposed in [2] that is justified from a theoretical point of view in the linear case. In $[8,17]$, an all Mach correction is proposed that allows to obtain an accurate scheme at low Mach number and that exactly matches the Godunov scheme when the Mach number is greater than one. In [5], this all Mach correction is justified from a theoretical point of view in the linear case. However, these previous works do not take the porosity into account, while it is a key ingredient to simulate the flow in a nuclear reactor core. Indeed, there is some section reduction (porosity) in a nuclear reactor core. Moreover, the flow may be diphasic in the core and the volumic fraction of vapor (or liquid) - that characterizes the diphasic state of the flow - may be considered as a porosity (from a mathematical point of view).

In this work, we introduce the porosity in our model and we study the behaviour of the Godunov scheme at low Mach number on a cartesian mesh by using the modified equation associated to the numerical scheme. It turns out that the numerical problems that occur at low Mach number for the barotropic Euler system with porosity are due to the fact that the kernel of the Godunov scheme applied to the linear wave equation with porosity is different from the stationary space of the continuous equation because of the numerical diffusion. This theoretical explanation is identical to the one proposed without porosity. Nevertheless, we have to introduce some theoretical tools taking the porosity into account to obtain this result (e.g. generalization of the Hodge decomposition to a weighted space where the weight is the porosity). This allows us to also propose and justify a low Mach correction and an all Mach correction that restore the accuracy of the scheme at low Mach number for the linear wave equation with porosity.

\section{LINEAR WAVE EQUATION WITH POROSITY}

The dimensionless barotropic Euler system with porosity may be written as

$$
\left\{\begin{array}{l}
\partial_{t}(\alpha \rho)+\nabla \cdot(\alpha \rho \mathbf{u})=0, \\
\partial_{t}(\alpha \rho \mathbf{u})+\nabla \cdot(\alpha \rho \mathbf{u} \otimes \mathbf{u})+\frac{\alpha}{M^{2}} \nabla p=0
\end{array}\right.
$$

In (1), $M$ is the Mach number that is supposed to be small and $\alpha(\mathbf{x})$ is the porosity, $t \geq 0$ and $\mathbf{x} \in \Omega$ are respectively the time and space variables. The quantities $\rho, \mathbf{u}$ and $p(\rho)$ are respectively the density, the velocity field and the pressure law of the fluid. We assume that the pressure law satisfies $p^{\prime}(\rho)>0$ and that $\alpha(\mathbf{x})$ is a known function that takes its values in $\left[\alpha_{\min }, 1\right]$, where $\alpha_{\min }>0$ is a constant which does not depend on $M$.

When the geometry is $2 \mathrm{D}$ or $3 \mathrm{D}$ and the cells of the mesh are not triangular (in 2D) nor tetrahedral (in 3D), finite volume Godunov type schemes applied to (1) with periodic boundary conditions are known to be inaccurate at low Mach number when $\nabla \alpha=0[2,5,7]$, contrarily to staggered schemes on cartesian meshes [7,15]. To better understand this behavior when $\nabla \alpha \neq 0$ and to propose a low Mach correction (when it is necessary) and an all Mach correction (when we want to recover the Godunov scheme for Mach numbers of order one) in the spirit of what is done in [5], we introduce tools adapted to a linearization of (1) around $\left(\rho=\rho_{\star}, \mathbf{u}=0\right)$ when $\Omega$ is periodic and we extend to the $2 \mathrm{D} / 3 \mathrm{D}$ case some $1 \mathrm{D}$ results proposed in [6]. For this purpose, we set the reference sound speed to $\frac{a_{\star}}{M}\left(a_{\star}^{2}=p^{\prime}\left(\rho_{\star}\right)\right)$ and we define $r(t, \mathbf{x})$ such as

$$
\rho(t, \mathbf{x}):=\rho_{\star}\left(1+\frac{M}{a_{\star}} r(t, \mathbf{x})\right)
$$


where formally $\frac{M}{a_{\star}} r \ll 1$. By injecting (2) in (1), we obtain the system

$$
\left\{\begin{array}{l}
\partial_{t}(\alpha r)+\nabla \cdot(\alpha r \mathbf{u})+\frac{a_{\star}}{M} \nabla \cdot(\alpha \mathbf{u})=0 \\
\partial_{t}(\alpha \mathbf{u})+(\alpha \mathbf{u} \cdot \nabla) \mathbf{u}+\frac{\alpha}{M} \frac{p^{\prime}\left(\rho_{\star}\left(1+\frac{M}{a_{\star}} r\right)\right)}{a_{\star}\left(1+\frac{M}{a_{\star}} r\right)} \nabla r=0 .
\end{array}\right.
$$

By linearizing around $(r, \mathbf{u})=(0,0)$, we obtain the linear wave equation with porosity

$$
\partial_{t}(\alpha q)+\frac{L_{\alpha}}{M}(q)=0 \quad \text { where } \quad q=\left(\begin{array}{c}
r \\
\mathbf{u}
\end{array}\right) \quad \text { and } \quad L_{\alpha}(q)=a_{\star}\left(\begin{array}{c}
\nabla \cdot(\alpha \mathbf{u}) \\
\alpha \nabla r
\end{array}\right)
$$

where $a_{\star} / M$ is the sound speed ( $a_{\star}$ is of order 1 and $M \ll 1$ at low Mach number).

\section{Weighted SPACES $\mathcal{E}_{\alpha}$ AND $\mathcal{E}_{\alpha}^{\perp}$}

We are interested in the properties of System (3) solved on a torus $\mathbb{T} \subset \mathbb{R}^{d \in\{1,2,3\}}$ (that is to say with periodic boundary conditions). For this, we assume that $\alpha$ is a periodic function on $\mathbb{T}$ and we define the weighted Hilbert space

$$
L_{\alpha}^{2}(\mathbb{T})^{1+d}:=\left\{q:=\left.(r, \mathbf{u})^{T}\left|\int_{\mathbb{T}} r^{2} \alpha d x+\int_{\mathbb{T}}\right| \mathbf{u}\right|^{2} \alpha d x<+\infty\right\}
$$

endowed with the scalar product

$$
\left\langle q_{1}, q_{2}\right\rangle_{\alpha}=\int_{\mathbb{T}} r_{1} r_{2} \alpha d x+\int_{\mathbb{T}} \mathbf{u}_{1} \cdot \mathbf{u}_{2} \alpha d x
$$

Of course, the space $L_{\alpha}^{2}$ must not be confused with the acoustic operator $L_{\alpha}$. We use the same notation to define the spaces $H_{\alpha}^{1}(\mathbb{T})$ and $H_{\alpha}^{2}(\mathbb{T})$ that are generalizations of $H^{1}(\mathbb{T})$ and $H^{2}(\mathbb{T})$ to weighted spaces. We note that since $\alpha(\mathbf{x}) \in\left[\alpha_{\min }, 1\right]$ with $\alpha_{\min }>0$, the functions $\alpha$ and $\frac{1}{\alpha}$ are in $L^{\infty}(\mathbb{T})$, and we have $L_{\alpha}^{2}(\mathbb{T})=L^{2}(\mathbb{T})$, $H_{\alpha}^{1}(\mathbb{T})=H^{1}(\mathbb{T})$ and $H_{\alpha}^{2}(\mathbb{T})=H^{2}(\mathbb{T})$. Nevertheless, we keep the index $\alpha$ to define these spaces to refer to the scalar product (4). At last, we define the space

$$
\mathcal{E}_{\alpha}:=\left\{q=(r, \mathbf{u})^{T} \in L_{\alpha}^{2}(\mathbb{T})^{1+d} \mid \nabla r=0 \text { and } \nabla \cdot(\alpha \mathbf{u})=0\right\}=\operatorname{Ker} L_{\alpha} .
$$

When $\alpha=1, \mathcal{E}_{\alpha}$ is named the incompressible space (see [2]). We have the following result:

Lemma 3.1. We have

$$
\begin{aligned}
& \mathcal{E}_{\alpha}^{\perp}=\left\{q=(r, \boldsymbol{u})^{T} \in L_{\alpha}^{2}(\mathbb{T})^{1+d} \mid \int_{\mathbb{T}} r \alpha d x=0 \text { and } \exists \phi \in H_{\alpha}^{1}(\mathbb{T}), \boldsymbol{u}=\nabla \phi\right\}, \\
& \mathcal{E}_{\alpha} \oplus \mathcal{E}_{\alpha}^{\perp}=L_{\alpha}^{2}(\mathbb{T})^{1+d} .
\end{aligned}
$$

In other words, any $q=(r, \boldsymbol{u})^{T} \in L_{\alpha}^{2}(\mathbb{T})^{1+d}$ can be decomposed into

$$
q=\hat{q}+q^{\perp}
$$

where $\hat{q}=(\hat{r}, \hat{\boldsymbol{u}})^{T} \in \mathcal{E}_{\alpha}$ and $q^{\perp}=\left(r^{\perp}, \boldsymbol{u}^{\perp}\right)^{T} \in \mathcal{E}_{\alpha}^{\perp}$, this decomposition is unique and orthogonal with respect to the scalar product defined by (4). 
We call $\mathcal{E}_{\alpha}^{\perp}$ the acoustic space. This is a generalization of the Hodge decomposition to the weighted space $L_{\alpha}^{2}(\mathbb{T})^{1+d}$. The decomposition (7) defines the orthogonal projection

$$
\begin{aligned}
\mathbb{P}_{\alpha}: L_{\alpha}^{2}(\mathbb{T})^{1+d} & \longrightarrow \mathcal{E}_{\alpha} \\
q & \longmapsto \mathbb{P}_{\alpha} q:=\hat{q} .
\end{aligned}
$$

Proof. We firstly prove (5). We note $A$ the space

$$
A:=\left\{q=(r, \mathbf{u})^{T} \in L_{\alpha}^{2}(\mathbb{T})^{1+d} \mid \int_{\mathbb{T}} r \alpha d x=0 \text { and } \exists \phi \in H_{\alpha}^{1}(\mathbb{T}), \mathbf{u}=\nabla \phi\right\} .
$$

Firstly, we prove that $A \subset \mathcal{E}_{\alpha}^{\perp}$ and, secondly, we prove that $\mathcal{E}_{\alpha}^{\perp} \subset A$. Let $q_{1}=\left(r_{1}, \mathbf{u}_{1}\right)^{T} \in A$. For all $q_{2}=$ $\left(r_{2}, \mathbf{u}_{2}\right)^{T} \in \mathcal{E}_{\alpha}$, we have

$$
\begin{aligned}
\left\langle q_{1}, q_{2}\right\rangle_{\alpha} & =\int_{\mathbb{T}} r_{1} r_{2} \alpha d x+\int_{\mathbb{T}} \mathbf{u}_{1} \cdot \mathbf{u}_{2} \alpha d x=r_{2} \int_{\mathbb{T}} r_{1} \alpha d x+\int_{\mathbb{T}} \nabla \phi_{1} \cdot \mathbf{u}_{2} \alpha d x \\
& =0+\int_{\partial \mathbb{T}} \phi_{1}\left(\alpha \mathbf{u}_{2}\right) \cdot \mathbf{n} d \sigma-\int_{\mathbb{T}} \phi_{1} \nabla \cdot\left(\alpha \mathbf{u}_{2}\right) d x=\int_{\partial \mathbb{T}} \phi_{1}\left(\alpha \mathbf{u}_{2}\right) \cdot \mathbf{n} d \sigma=0
\end{aligned}
$$

because $q_{2} \in \mathcal{E}_{\alpha}$ and $\phi_{1}\left(\alpha \mathbf{u}_{2}\right)$ is periodic. This proves that $A \subset \mathcal{E}_{\alpha}^{\perp}$. Let $q_{1}=\left(r_{1}, \mathbf{u}_{1}\right)^{T} \in \mathcal{E}_{\alpha}^{\perp}$. For all $q_{2}=\left(r_{2}, \mathbf{u}_{2}\right)^{T} \in \mathcal{E}_{\alpha}$, we have

$$
\left\langle q_{1}, q_{2}\right\rangle_{\alpha}=0 \Longrightarrow \int_{\mathbb{T}} r_{1} r_{2} \alpha d x+\int_{\mathbb{T}} \mathbf{u}_{1} \cdot \mathbf{u}_{2} \alpha d x=0 \quad \Longrightarrow \quad r_{2} \int_{\mathbb{T}} r_{1} \alpha d x+\int_{\mathbb{T}} \mathbf{u}_{1} \cdot\left(\alpha \mathbf{u}_{2}\right) d x=0 .
$$

Then $\int_{\mathbb{T}} r_{1} \alpha d x=0$ and $\int_{\mathbb{T}} \mathbf{u}_{1} \cdot\left(\alpha \mathbf{u}_{2}\right) d x=0$ for all $\mathbf{u}_{2} \in L_{\alpha}^{2}(\mathbb{T})^{d}$ such that $\nabla \cdot\left(\alpha \mathbf{u}_{2}\right)=0$. Moreover, since $\alpha$ and $\frac{1}{\alpha}$ are in $L^{\infty}(\mathbb{T})$, the last equality is equivalent to $\int_{\mathbb{T}} \mathbf{u}_{1} \cdot \tilde{\mathbf{u}}_{2} d x=0$ for all $\tilde{\mathbf{u}}_{2} \in \mathcal{E}:=\left\{\mathbf{u} \in L^{2}(\mathbb{T})^{d} \mid \nabla \cdot \mathbf{u}=0\right\}$. Therefore, $\mathbf{u}_{1} \in \mathcal{E}^{\perp}$ for the classical $L^{2}(\mathbb{T})^{d}$ scalar product. It is a classical result that

$$
\mathcal{E}^{\perp}=\left\{\mathbf{u} \in L^{2}(\mathbb{T})^{d} \mid \exists \phi \in H^{1}(\mathbb{T}), \mathbf{u}=\nabla \phi\right\}
$$

This implies that $\exists \phi_{1} \in H_{\alpha}^{1}(\mathbb{T})$ such that $\mathbf{u}_{1}=\nabla \phi_{1}$, which allows to write that $\mathcal{E}_{\alpha}^{\perp} \subset A$. To conclude, we have $\mathcal{E}_{\alpha}^{\perp}=A$.

Now, we prove (6). Since the inclusion $\subset$ is trivial, we just have to prove that $\mathcal{E}_{\alpha} \oplus \mathcal{E}_{\alpha}^{\perp} \supset L_{\alpha}^{2}(\mathbb{T})^{1+d}$. Let $q=(r, \mathbf{u})^{T} \in L_{\alpha}^{2}(\mathbb{T})^{1+d}$. We dissociate the construction of $\left(\hat{r}, r^{\perp}\right)$ from that of $\left(\hat{\mathbf{u}}, \mathbf{u}^{\perp}\right)$. For $r$, we can define $\hat{r}=\frac{1}{\int_{\mathbb{T}} \alpha d x} \int_{\mathbb{T}} r \alpha d x$ since $\alpha \geq \alpha_{\min }>0$ implies that $\int_{\mathbb{T}} \alpha d \mathbf{x}>0$. Moreover, $\left.\left.\alpha \in\right] 0,1\right]$ implies that $\int_{\mathbb{T}} \alpha d \mathbf{x} \leq$ $\|\alpha\|_{\infty}|\mathbb{T}|<+\infty$ and with the Cauchy-Schwarz inequality, we obtain

$$
\left|\int_{\mathbb{T}} r \alpha d \mathbf{x}\right| \leq\left(\int_{\mathbb{T}} r^{2} \alpha d \mathbf{x}\right)^{\frac{1}{2}}\left(\int_{\mathbb{T}} \alpha d \mathbf{x}\right)^{\frac{1}{2}} \leq\|r\|_{L_{\alpha}^{2}}\|\alpha\|_{\infty}^{\frac{1}{2}}|\mathbb{T}|^{\frac{1}{2}}<+\infty .
$$

Then, since

$$
\int_{\mathbb{T}}(r-\hat{r}) \alpha d x=\int_{\mathbb{T}} r \alpha d x-\hat{r} \int_{\mathbb{T}} \alpha d x=0
$$

we can write $r=\hat{r}+(r-\hat{r})$ with $\nabla \hat{r}=0$ and $\int_{\mathbb{T}}(r-\hat{r}) \alpha d x=0$ which gives the decomposition for $r$. For $\mathbf{u}$, the construction is slightly more difficult. We want to construct $\hat{\mathbf{u}}$ and $\nabla \phi$ such that $\mathbf{u}=\hat{\mathbf{u}}+\nabla \phi$ with $\nabla \cdot(\alpha \hat{\mathbf{u}})=0$. 
It is then sufficient to prove that there exists $\phi \in H_{\alpha}^{1}(\mathbb{T})$ such that

$$
\left\{\begin{array}{l}
\nabla \cdot(\alpha \nabla \phi)=\nabla \cdot(\alpha \mathbf{u}), \\
\int_{\mathbb{T}} \phi \alpha d x=0
\end{array}\right.
$$

and to set $\hat{\mathbf{u}}=\mathbf{u}-\nabla \phi$. We note $H_{\alpha, 0}^{1}(\mathbb{T}) \subset H_{\alpha}^{1}(\mathbb{T})$ the subset of functions $\phi$ such that $\int_{\mathbb{T}} \phi \alpha d x=0$. We write (9) under variational form:

Find $\phi \in H_{\alpha, 0}^{1}(\mathbb{T}) \quad$ such that $\quad \forall \psi \in H_{\alpha, 0}^{1}(\mathbb{T}): \quad a(\phi, \psi):=\int_{\mathbb{T}}(\alpha \nabla \phi) \cdot \nabla \psi d \mathbf{x}=\int_{\mathbb{T}}(\alpha \mathbf{u}) \cdot \nabla \psi d \mathbf{x}=: L(\psi)$

Moreover, $L$ is a continuous linear functional on the Hilbert space $H_{\alpha, 0}^{1}(\mathbb{T})$ because

$$
|L(\psi)|=\left|\int_{\mathbb{T}} \sqrt{\alpha} \mathbf{u} \cdot \sqrt{\alpha} \nabla \psi d \mathbf{x}\right| \leq\|\mathbf{u}\|_{L_{\alpha}^{2}}\|\nabla \psi\|_{L_{\alpha}^{2}} \leq\|\mathbf{u}\|_{L_{\alpha}^{2}}\|\psi\|_{H_{\alpha}^{1}}
$$

By using a similar argument, we also prove that $a(\cdot, \cdot)$ is a symmetric bilinear form that is continuous on $H_{\alpha, 0}^{1}(\mathbb{T})$. To prove the coercivity of $a$, we use a generalization of the Poincaré-Wirtinger inequality to the probabilistic measure $\mu:=\frac{\alpha}{\int_{\mathbb{T}} \alpha d \mathbf{x}}$ on the convex space $\mathbb{T}$ (see Appendix). As $\mu$ and $\frac{1}{\mu}$ are in $L^{\infty}(\mathbb{T})$, for $\phi \in H_{\alpha}^{1}(\mathbb{T})$ and $\bar{\phi}:=\frac{1}{\int_{\mathbb{T}} \alpha d \mathbf{x}} \int_{\mathbb{T}} \phi \alpha d \mathbf{x}$, we have

$$
\int_{\mathbb{T}}|\phi-\bar{\phi}|^{2} \mu d \mathbf{x} \leq 2 \operatorname{diam}(\mathbb{T})^{2}\|\mu\|_{\infty}\left\|\frac{1}{\mu}\right\|_{\infty} \int_{\mathbb{T}}|\nabla \phi|^{2} \mu d \mathbf{x}
$$

where $\operatorname{diam}(\mathbb{T}):=\sup _{(x, y) \in \mathbb{T}^{2}}|x-y|$, which is equivalent to $\int_{\mathbb{T}}|\phi-\bar{\phi}|^{2} \alpha d \mathbf{x} \leq 2 \operatorname{diam}(\mathbb{T})^{2}\|\alpha\|_{\infty}\left\|\frac{1}{\alpha}\right\|_{\infty} \int_{\mathbb{T}}|\nabla \phi|^{2} \alpha d \mathbf{x}$. Thus, we can write that

$$
C_{\alpha}(\mathbb{T}) \int_{\mathbb{T}}|\phi-\bar{\phi}|^{2} \alpha d \mathbf{x} \leq \int_{\mathbb{T}}|\nabla \phi|^{2} \alpha d \mathbf{x}
$$

with $C_{\alpha}(\mathbb{T}):=\frac{1}{2 \operatorname{diam}(\mathbb{T})^{2}\|\alpha\|_{\infty}\left\|\frac{1}{\alpha}\right\|_{\infty}}>0$. For $\phi \in H_{\alpha, 0}^{1}(\mathbb{T})$, we have $\bar{\phi}=0$ and we can write

$$
\begin{aligned}
a(\phi, \phi) & =\int_{\mathbb{T}}|\nabla \phi|^{2} \alpha d \mathbf{x}=\frac{1}{2} \int_{\mathbb{T}}|\nabla \phi|^{2} \alpha d \mathbf{x}+\frac{1}{2} \int_{\mathbb{T}}|\nabla \phi|^{2} \alpha d \mathbf{x} \\
& \geq \frac{1}{2} \int_{\mathbb{T}}|\nabla \phi|^{2} \alpha d \mathbf{x}+\frac{C_{\alpha}(\mathbb{T})}{2} \int_{\mathbb{T}}|\phi|^{2} \alpha d \mathbf{x} \geq \frac{1}{2} \min \left(1, C_{\alpha}(\mathbb{T})\right)\|\phi\|_{H_{\alpha}^{1}}^{2}
\end{aligned}
$$

which means that $a(\cdot, \cdot)$ is coercive. Then, by applying the Lax-Milgram theorem, we obtain the existence of a unique function $\phi$ in $H_{\alpha, 0}^{1}(\mathbb{T})$ such that $\forall \psi \in H_{\alpha, 0}^{1}(\mathbb{T}), a(\phi, \psi)=L(\psi)$ that is to say

$$
\forall \psi \in H_{\alpha, 0}^{1}(\mathbb{T}): \quad \int_{\mathbb{T}}(\alpha \nabla \phi) \cdot \nabla \psi d \mathbf{x}=\int_{\mathbb{T}}(\alpha \mathbf{u}) \cdot \nabla \psi d \mathbf{x}
$$


We note $D(\mathbb{T})$ the set of functions $C^{\infty}(\mathbb{T})$ with a compact support. For all $\psi$ in $D(\mathbb{T})$, the function

$$
\tilde{\psi}:=\psi-\bar{\psi}=\psi-\frac{\int_{\mathbb{T}} \psi \alpha d \mathbf{x}}{\int_{\mathbb{T}} \alpha d \mathbf{x}}
$$

is in $H_{\alpha, 0}^{1}(\mathbb{T})$. Then, $\tilde{\psi}$ satisfies (12). And since $\nabla \tilde{\psi}=\nabla \psi$, we have

$$
\forall \psi \in D(\mathbb{T}): \quad \int_{\mathbb{T}}(\alpha \nabla \phi) \cdot \nabla \psi d \mathbf{x}=\int_{\mathbb{T}}(\alpha \mathbf{u}) \cdot \nabla \psi d \mathbf{x}
$$

that is to say

$$
\forall \psi \in D(\mathbb{T}): \quad\langle-\nabla \cdot(\alpha \nabla \phi), \psi\rangle_{D, D^{\prime}}=\langle-\nabla \cdot(\alpha \mathbf{u}), \psi\rangle_{D, D^{\prime}}
$$

In other words, $\nabla \cdot(\alpha \nabla \phi)=\nabla \cdot(\alpha \mathbf{u})$ in the sense of distribution. By setting $\hat{\mathbf{u}}=\mathbf{u}-\nabla \phi$, we obtain $\mathbf{u}=\hat{\mathbf{u}}+\nabla \phi$ with $\nabla \cdot(\alpha \hat{\mathbf{u}})=0$. Thus $L_{\alpha}^{2}(\mathbb{T})^{1+d} \subset \mathcal{E}_{\alpha} \oplus \mathcal{E}_{\alpha}^{\perp}$.

\section{Properties of the linear Wave equation With Porosity}

We now detail some properties of the linear wave equation with porosity. These properties will not be always satisfied in the discrete case.

Lemma 4.1. Let $q(t, \boldsymbol{x})$ be the solution of (3) on $\mathbb{T} \subset \mathbb{R}^{d \in\{1,2,3\}}$ with initial condition $q^{0}$. Then:

1) $\forall q^{0} \in \mathcal{E}_{\alpha}: q(t \geq 0) \in \mathcal{E}_{\alpha}$.

2) $\forall q^{0} \in \mathcal{E}_{\alpha}^{\perp}: q(t \geq 0) \in \mathcal{E}_{\alpha}^{\perp}$.

Proof. The first point is a direct consequence of the expression of System (3) because $\mathcal{E}_{\alpha}=K e r L_{\alpha}$ and then for all $t \geq 0, q(t)=q^{0}$. Let $q^{0}=\left(r^{0}, \mathbf{u}^{0}\right)^{T} \in \mathcal{E}_{\alpha}^{\perp}$. We have $\alpha q(t)=\alpha q^{0}-\frac{1}{M} \int_{0}^{t} L_{\alpha}(q) d \tau$. Then, for all $\tilde{q}=(\tilde{r}, \tilde{\mathbf{u}})^{T} \in \mathcal{E}_{\alpha}$, we have

$$
\langle q, \tilde{q}\rangle_{\alpha}=\left\langle q^{0}, \tilde{q}\right\rangle_{\alpha}-\frac{1}{M} \int_{0}^{t} \int_{\mathbb{T}} L_{\alpha}(q) \cdot \tilde{q} d \mathbf{x} d \tau
$$

with

$$
\begin{aligned}
\int_{\mathbb{T}} L_{\alpha}(q) \cdot \tilde{q} d \mathbf{x} & =a_{\star} \int_{\mathbb{T}}(\nabla \cdot(\alpha \mathbf{u}) \tilde{r}+\alpha \nabla r \cdot \tilde{\mathbf{u}}) d \mathbf{x}=-a_{\star} \int_{\mathbb{T}}((\alpha \mathbf{u}) \cdot \nabla \tilde{r}+r \nabla \cdot(\alpha \tilde{\mathbf{u}})) d \mathbf{x} \\
& =-\int_{\mathbb{T}} q \cdot L_{\alpha}(\tilde{q}) d \mathbf{x}=0
\end{aligned}
$$

because $\tilde{q} \in \mathcal{E}_{\alpha}=\operatorname{Ker} L_{\alpha}$. Then, for all $\tilde{q}=(\tilde{r}, \tilde{\mathbf{u}})^{T} \in \mathcal{E}_{\alpha},\langle q, \tilde{q}\rangle_{\alpha}=\left\langle q^{0}, \tilde{q}\right\rangle_{\alpha}=0$ which means that $q \in \mathcal{E}_{\alpha}^{\perp}$.

For all $q \in L_{\alpha}^{2}(\mathbb{T})^{1+d}$, we now define the energy $E_{\alpha}:=\langle q, q\rangle_{\alpha}$. The following lemma is an extension of the energy conservation property of the classical linear wave equation:

Lemma 4.2. Let $q(t, \boldsymbol{x})$ be the solution of (3) on $\mathbb{T} \subset \mathbb{R}^{d \in\{1,2,3\}}$. Then:

$$
\forall t \geq 0: \quad E_{\alpha}(t \geq 0)=E_{\alpha}(t=0) .
$$


Proof. For a solution $q=(r, \mathbf{u})^{T}$ of System (3), we have

$$
\partial_{t}(\alpha q)+\frac{L_{\alpha}}{M}(q)=0 \Rightarrow \frac{1}{2} \frac{d}{d t}\langle q, q\rangle_{\alpha}+\left\langle\frac{q}{\alpha}, \frac{L_{\alpha}}{M}(q)\right\rangle_{\alpha}=0 \Rightarrow \frac{d}{d t} E_{\alpha}(t)=0
$$

because

$$
\left\langle\frac{q}{\alpha}, \frac{L_{\alpha}}{M}(q)\right\rangle_{\alpha}=\frac{a_{\star}}{M} \int_{\mathbb{T}}\left(\frac{r}{\alpha} \nabla \cdot(\alpha \mathbf{u})+\frac{\mathbf{u}}{\alpha} \cdot(\alpha \nabla r)\right) \alpha d \mathbf{x}=\frac{a_{\star}}{M} \int_{\partial \mathbb{T}} r(\alpha \mathbf{u}) \cdot \mathbf{n} d \sigma=0
$$

by using the periodicity of $\mathbb{T}$.

\section{Godunov SCHEME With POROSity}

We construct the Godunov scheme with porosity.

\subsection{Finite volume scheme and Riemann problem}

Let us suppose that the domain $\mathbb{T} \subset \mathbb{R}^{d \in\{1,2,3\}}$ is discretized by $N$ cells $\Omega_{i}$. Let $\Gamma_{i j}$ be the common edge (in $2 \mathrm{D}$ and common face in $3 \mathrm{D}$ ) of the two neighboring cells $\Omega_{i}$ and $\Omega_{j}$ and $\mathbf{n}_{i j}$ the unit vector normal to $\Gamma_{i j}$ pointing from $\Omega_{i}$ to $\Omega_{j}$. We assume that the quantities $(\alpha, \alpha r, \alpha \mathbf{u})$ are defined on the cells $\Omega_{i}$ by

$$
\alpha_{i}=\frac{1}{\left|\Omega_{i}\right|} \int_{\Omega_{i}} \alpha d \mathbf{x}, \quad(\alpha r)_{i}=\frac{1}{\left|\Omega_{i}\right|} \int_{\Omega_{i}} r \alpha d \mathbf{x} \quad(\alpha \mathbf{u})_{i}=\frac{1}{\left|\Omega_{i}\right|} \int_{\Omega_{i}} \alpha \mathbf{u} d \mathbf{x} .
$$

After integration of (3) over each $\Omega_{i}$, the semi-discrete finite volume scheme applied to the resolution of the linear wave equation with porosity is given by

$$
\left\{\begin{array}{l}
\frac{d}{d t}(\alpha r)_{i}+\frac{a_{\star}}{M} \frac{1}{\left|\Omega_{i}\right|} \sum_{\Gamma_{i j} \subset \partial \Omega_{i}}\left|\Gamma_{i j}\right|(\alpha \mathbf{u} \cdot \mathbf{n})_{i j}=0, \\
\frac{d}{d t}(\alpha \mathbf{u})_{i}+\frac{a_{\star}}{M} \frac{\alpha_{i}}{\left|\Omega_{i}\right|} \sum_{\Gamma_{i j} \subset \partial \Omega_{i}}\left|\Gamma_{i j}\right| r_{i j} \mathbf{n}_{i j}=0 .
\end{array}\right.
$$

The Godunov approach consists in defining $\left(r_{i j},(\alpha \mathbf{u} \cdot \mathbf{n})_{i j}\right)$ as the solution of a $1 D$ Riemann problem on $\Gamma_{i j}$ in the $\mathbf{n}_{i j}$ direction. A $1 \mathrm{D}$ study performed in [6] has shown that it is better to work with the $(r, \alpha \mathbf{u})$ set of variables, and thus we propose to solve the following Riemann problem:

$$
\left\{\begin{array}{l}
\partial_{t} r_{\xi}+\frac{a_{\star}}{M} \frac{1}{\alpha_{i j}} \partial_{\xi}\left((\alpha u)_{\xi}\right)=0, \\
\partial_{t}\left((\alpha u)_{\xi}\right)+\frac{a_{\star}}{M} \alpha_{i j} \partial_{\xi} r_{\xi}=0, \\
\left(r_{\xi},(\alpha u)_{\xi}\right)(t=0, \xi)=\left\{\begin{array}{l}
\left(r_{i},(\alpha \mathbf{u})_{i} \cdot \mathbf{n}_{i j}\right) \text { if } \xi<0, \\
\left(r_{j},(\alpha \mathbf{u})_{j} \cdot \mathbf{n}_{i j}\right) \text { otherwise }
\end{array}\right.
\end{array}\right.
$$

where $\xi$ is the coordinate in the $\mathbf{n}_{i j}$ direction and $\alpha_{i j}$ is a representative value of $\alpha$ on $\Gamma_{i j}$. For example, since $\alpha$ is a known function, we may choose its mean value on $\Gamma_{i j}$; otherwise the mean value of $\alpha_{i}$ and $\alpha_{j}$, i.e. $\alpha_{i j}=\frac{\alpha_{i}+\alpha_{j}}{2}$ may also be used. Another point of view could be to consider $\alpha$ as an additional unknown of the system, satisfying $\partial_{t} \alpha=0$, and to solve the Riemann problem associated to this augmented system. However, the study of this scheme is out of the scope of the present work. 


\subsection{Solution of the Riemann problem}

We explicit the solution of the Riemann problem (15). By a simple scaling argument, the solution of (15) is a function only of $\xi / t$. We set $U=(r, J)^{T}$ where $J=\alpha u$ and we write (15) under the form

$$
\partial_{t} U+A \partial_{\xi} U=0, \quad \text { where } \quad A=\left[\begin{array}{cc}
0 & \frac{a_{\star}}{M \alpha_{i j}} \\
\frac{a_{\star} \alpha_{i j}}{M} & 0
\end{array}\right] .
$$

System (15) is hyperbolic and the matrix $A$ admits the two distinct eigenvalues $\lambda_{1}=-\frac{a_{\star}}{M}<\lambda_{2}=\frac{a_{\star}}{M}$. The solution $R\left(U_{i}, U_{j}, \xi / t\right)$ of (15) is under the form

$$
R\left(U_{i}, U_{j}, \xi / t\right)=\left\{\begin{array}{l}
U_{i}, \text { if } \xi / t<\lambda_{1}, \\
U^{\star}, \text { if } \lambda_{1}<\xi / t<\lambda_{2}, \\
U_{j}, \text { if } \xi / t>\lambda_{2},
\end{array}\right.
$$

with

$$
U_{i}:=\left(r_{i},(\alpha \mathbf{u})_{i} \cdot \mathbf{n}_{i j}\right) \quad \text { and } \quad U_{j}:=\left(r_{j},(\alpha \mathbf{u})_{j} \cdot \mathbf{n}_{i j}\right)
$$

and where we have to find $U^{\star}$. We use the Riemann invariants to explicit $U^{\star}=\left(r^{\star}, J^{\star}\right)^{T}$. We can prove that $\mathbf{v}_{1}=\left(1,-\alpha_{i j}\right)^{T}$ (resp. $\left.\mathbf{v}_{2}=\left(1, \alpha_{i j}\right)^{T}\right)$ is an eigenvector of $A$ associated to $\lambda_{1}$ (resp. $\left.\lambda_{2}\right)$ and that $R_{1}=J+\alpha_{i j} r$ is a 1-Riemann invariant and $R_{2}=J-\alpha_{i j} r$ is a 2-Riemann invariant. As a Riemann invariant is constant through a linearly degenerate wave, we obtain

$$
\left\{\begin{array} { l } 
{ J ^ { \star } + \alpha _ { i j } r ^ { \star } = J _ { i } + \alpha _ { i j } r _ { i } , } \\
{ J ^ { \star } - \alpha _ { i j } r ^ { \star } = J _ { j } - \alpha _ { i j } r _ { j } }
\end{array} \Longrightarrow \left\{\begin{array}{l}
r^{\star}=\frac{r_{i}+r_{j}}{2}+\frac{1}{2 \alpha_{i j}}\left(J_{i}-J_{j}\right), \\
J^{\star}=\frac{J_{i}+J_{j}}{2}+\frac{\alpha_{i j}}{2}\left(r_{i}-r_{j}\right) .
\end{array}\right.\right.
$$

\subsection{The Godunov scheme}

Finally, setting in (14) $r_{i j}=r^{\star}$ and $(\alpha \mathbf{u} \cdot \mathbf{n})_{i j}=J^{\star}$ given by (16), the Godunov scheme is given by

$$
\left\{\begin{array}{l}
\frac{d}{d t}(\alpha r)_{i}+\frac{a_{\star}}{2 M} \frac{1}{\left|\Omega_{i}\right|} \sum_{\Gamma_{i j} \subset \partial \Omega_{i}}\left|\Gamma_{i j}\right|\left[\left((\alpha \mathbf{u})_{i}+(\alpha \mathbf{u})_{j}\right) \cdot \mathbf{n}_{i j}+\alpha_{i j}\left(r_{i}-r_{j}\right)\right]=0, \\
\frac{d}{d t}(\alpha \mathbf{u})_{i}+\frac{a_{\star}}{2 M} \frac{\alpha_{i}}{\left|\Omega_{i}\right|} \sum_{\Gamma_{i j} \subset \partial \Omega_{i}}\left|\Gamma_{i j}\right|\left[r_{i}+r_{j}+\frac{\kappa}{\alpha_{i j}}\left((\alpha \mathbf{u})_{i}-(\alpha \mathbf{u})_{j}\right) \cdot \mathbf{n}_{i j}\right] \mathbf{n}_{i j}=0
\end{array}\right.
$$

where $\kappa=1$. We introduce the parameter $\kappa$ because this parameter will be important in the sequel.

\section{KeRnel of the FiRst ORDER MODIFIED EQUATION ON A CARTESIAN MESH}

To understand the behaviour of the Godunov scheme at low Mach number, a first step is to study the kernel of the spatial operator associated to the modified equation related to the Godunov scheme. Indeed, we will see that this kernel is strictly included in the kernel of the acoustic operator in (3) which is exactly equal to $\mathcal{E}_{\alpha}$. As a consequence, the Godunov scheme does not preserve some states in $\mathcal{E}_{\alpha}$. 


\subsection{First order modified equation on a cartesian mesh}

We suppose for the sake of simplicity that the space dimension is 2. Assume that the mesh is cartesian with the space step $\Delta x$ (resp. $\Delta y)$ in the $x$ (resp. y) direction. The subscript $(i, j)$ defines the center of each cell of the cartesian mesh, $\left(i \pm \frac{1}{2}, j\right)$ and $\left(i, j \pm \frac{1}{2}\right)$ defining the interfaces of the cell $(i, j)$. The Godunov scheme (17) can be written with

$$
\begin{array}{r}
\frac{d}{d t}(\alpha r)_{i, j}+\frac{a_{\star}}{M} \frac{\left(\alpha u_{x}\right)_{i+1, j}-\left(\alpha u_{x}\right)_{i-1, j}}{2 \Delta x}+\frac{a_{\star}}{M} \frac{\left(\alpha u_{y}\right)_{i, j+1}-\left(\alpha u_{y}\right)_{i, j-1}}{2 \Delta y} \\
=\frac{a_{\star}}{2 M \Delta x}\left(\alpha_{i+\frac{1}{2}, j}\left(r_{i+1, j}-r_{i, j}\right)-\alpha_{i-\frac{1}{2}, j}\left(r_{i, j}-r_{i-1, j}\right)\right) \\
+\frac{a_{\star}}{2 M \Delta y}\left(\alpha_{i, j+\frac{1}{2}}\left(r_{i, j+1}-r_{i, j}\right)-\alpha_{i, j-\frac{1}{2}}\left(r_{i, j}-r_{i, j-1}\right)\right), \\
\frac{d}{d t}\left(\alpha u_{x}\right)_{i, j}+\frac{a_{\star}}{M} \alpha_{i, j} \frac{r_{i+1, j}-r_{i-1, j}}{2 \Delta x}=\kappa \frac{a_{\star}}{2 M \Delta x} \alpha_{i, j}\left(\frac{1}{\alpha_{i+\frac{1}{2}, j}}\left(\left(\alpha u_{x}\right)_{i+1, j}-\left(\alpha u_{x}\right)_{i, j}\right)\right. \\
\left.-\frac{1}{\alpha_{i-\frac{1}{2}, j}}\left(\left(\alpha u_{x}\right)_{i, j}-\left(\alpha u_{x}\right)_{i-1, j}\right)\right) \\
\frac{d}{d t}\left(\alpha u_{y}\right)_{i, j}+\frac{a_{\star}}{M} \alpha_{i, j} \frac{r_{i, j+1}-r_{i, j-1}}{2 \Delta y}=\kappa \frac{a_{\star}}{2 M \Delta y} \alpha_{i, j}\left(\frac{1}{\alpha_{i, j+\frac{1}{2}}}\left(\left(\alpha u_{y}\right)_{i, j+1}-\left(\alpha u_{y}\right)_{i, j}\right)\right. \\
\left.-\frac{1}{\alpha_{i, j-\frac{1}{2}}}\left(\left(\alpha u_{y}\right)_{i, j}-\left(\alpha u_{y}\right)_{i, j-1}\right)\right)
\end{array}
$$

with $\kappa=1$. The first order modified equation associated to this scheme is given by

$$
\partial_{t}(\alpha q)+\frac{\mathcal{L}_{\kappa, \alpha}}{M}(q)=0
$$

where $\mathcal{L}_{\kappa, \alpha}=L_{\alpha}-M B_{\kappa, \alpha}$ with

$$
L_{\alpha}(q)=a_{\star}\left(\begin{array}{c}
\nabla \cdot(\alpha \mathbf{u}) \\
\alpha \nabla r
\end{array}\right) \quad \text { and } \quad B_{\kappa, \alpha}(q)=\left(\begin{array}{c}
\frac{a_{\star} \Delta x}{2 M} \partial_{x}\left(\alpha \partial_{x} r\right)+\frac{a_{\star} \Delta y}{2 M} \partial_{y}\left(\alpha \partial_{y} r\right) \\
\kappa \alpha \frac{a_{\star} \Delta x}{2 M} \partial_{x}\left(\frac{1}{\alpha} \partial_{x}\left(\alpha u_{x}\right)\right) \\
\kappa \alpha \frac{a_{\star} \Delta y}{2 M} \partial_{y}\left(\frac{1}{\alpha} \partial_{y}\left(\alpha u_{y}\right)\right)
\end{array}\right) .
$$

\subsection{Kernel of the modified equation and energy relation}

We study the kernel of the spatial operator associated to the modified equation (18). The structure of the kernel depends on the value of $\kappa$. The kernel for the Godunov scheme $(\kappa=1)$ is different from the incompressible space $\mathcal{E}_{\alpha}$. Indeed:

Lemma 6.1. (1) If $\kappa>0$, we have

$$
\operatorname{Ker} \mathcal{L}_{\kappa>0, \alpha}=\left\{q:=(r, \boldsymbol{u})^{T} \mid \nabla r=0 \text { and } \partial_{x}\left(\alpha u_{x}\right)=\partial_{y}\left(\alpha u_{y}\right)=0\right\} \subsetneq \mathcal{E}_{\alpha} .
$$

(2) If $\kappa=0$, we have

$$
\operatorname{Ker} \mathcal{L}_{\kappa=0, \alpha}=\mathcal{E}_{\alpha}
$$


Proof. If $\kappa=0$, we easily obtain that $\operatorname{Ker} \mathcal{L}_{\kappa=0, \alpha}=\mathcal{E}_{\alpha}$. Let us now suppose that $\kappa>0$. By using (13), we can write that

$$
\left\langle\frac{q}{\alpha}, \frac{\mathcal{L}_{\kappa, \alpha}}{M}(q)\right\rangle_{\alpha}=\left\langle\frac{q}{\alpha}, B_{\kappa, \alpha}(q)\right\rangle_{\alpha} .
$$

Let us choose $q:=(r, \mathbf{u})^{T} \in \operatorname{Ker} \mathcal{L}_{\kappa, \alpha}$. In that case, we deduce from the previous equality that

$$
\left\langle\frac{q}{\alpha}, B_{\kappa, \alpha}(q)\right\rangle_{\alpha}=0
$$

On the other hand, we have

$$
-\left\langle\frac{q}{\alpha}, B_{\kappa, \alpha}(q)\right\rangle_{\alpha}=\frac{a_{\star} \Delta x}{2 M}\left\|\partial_{x} r\right\|_{L_{\alpha}^{2}}^{2}+\frac{a_{\star} \Delta y}{2 M}\left\|\partial_{y} r\right\|_{L_{\alpha}^{2}}^{2}+\kappa \frac{a_{\star} \Delta x}{2 M}\left\|\frac{\partial_{x}\left(\alpha u_{x}\right)}{\alpha}\right\|_{L_{\alpha}^{2}}^{2}+\kappa \frac{a_{\star} \Delta y}{2 M}\left\|\frac{\partial_{y}\left(\alpha u_{y}\right)}{\alpha}\right\|_{L_{\alpha}^{2}}^{2} .
$$

This allows to write that

$$
\left\|\partial_{x} r\right\|_{L_{\alpha}^{2}}^{2}=\left\|\partial_{y} r\right\|_{L_{\alpha}^{2}}^{2}=\left\|\frac{\partial_{x}\left(\alpha u_{x}\right)}{\alpha}\right\|_{L_{\alpha}^{2}}^{2}=\left\|\frac{\partial_{y}\left(\alpha u_{y}\right)}{\alpha}\right\|_{L_{\alpha}^{2}}^{2}=0
$$

that is to say $\nabla r=0$ and $\partial_{x}\left(\alpha u_{x}\right)=\partial_{y}\left(\alpha u_{y}\right)=0$. This proves that $\operatorname{Ker} \mathcal{L}_{\kappa, \alpha} \subset \mathcal{A}$ with

$$
\mathcal{A}:=\left\{q:=(r, \mathbf{u})^{T} \mid \nabla r=0 \text { and } \partial_{x}\left(\alpha u_{x}\right)=\partial_{y}\left(\alpha u_{y}\right)=0\right\} .
$$

Let us now suppose that $q \in \mathcal{A}$. In that case, we have $q \in \mathcal{E}_{\alpha}=\operatorname{Ker} L_{\alpha}$ and $q \in \operatorname{Ker} B_{\kappa, \alpha}$ that is to say $q \in \operatorname{Ker}\left(L_{\alpha}-M B_{\kappa, \alpha}\right)=\operatorname{Ker} \mathcal{L}_{\kappa, \alpha}$. Thus, we have also $\mathcal{A} \subset \operatorname{Ker} \mathcal{L}_{\kappa, \alpha}$.

If the kernel depends on the value of $\kappa$, System (18) is dissipative for all $\kappa \geq 0$. Indeed:

Lemma 6.2. Let $q(t, \boldsymbol{x})$ be the solution of (18) on $\mathbb{T} \subset \mathbb{R}^{2}$. If $\kappa \geq 0$, System (18) is dissipative. That is to say:

$$
\forall t \geq 0: \quad \frac{d}{d t} E_{\alpha}(t) \leq 0 \quad \text { where } \quad E_{\alpha}(t):=\|q\|_{L_{\alpha}^{2}}^{2} .
$$

Proof. We have $\frac{1}{2} \frac{d}{d t} E_{\alpha}(t)=-\left\langle\frac{q}{\alpha}, \frac{\mathcal{L}_{\alpha, \kappa}}{M}(q)\right\rangle_{\alpha}$. And since $\left\langle\frac{q}{\alpha}, \frac{\mathcal{L}_{\alpha, \kappa}}{M}(q)\right\rangle_{\alpha}=-\left\langle\frac{q}{\alpha}, B_{\kappa, \alpha}(q)\right\rangle_{\alpha}$, we obtain by using (20)

$$
\frac{1}{2} \frac{d}{d t} E_{\alpha}(t)=-\frac{a_{\star}}{2 M}\left(\Delta x\left\|\partial_{x} r\right\|_{L_{\alpha}^{2}}^{2}+\Delta y\left\|\partial_{y} r\right\|_{L_{\alpha}^{2}}^{2}+\kappa \Delta x\left\|\frac{\partial_{x}\left(\alpha u_{x}\right)}{\alpha}\right\|_{L_{\alpha}^{2}}^{2}+\kappa \Delta y\left\|\frac{\partial_{y}\left(\alpha u_{y}\right)}{\alpha}\right\|_{L_{\alpha}^{2}}^{2}\right) .
$$

This equality allows to write that $\frac{d}{d t} E_{\alpha}(t) \leq 0$ for any $\kappa \geq 0$.

\section{Explanation of the inACCURACy of THE GOdunOV SCHEME ON A CARTESiAn MESH AT LOW MACH NUMBER BY USING THE MODIFIED EQUATION}

We studied the kernel $\operatorname{Ker} \mathcal{L}_{\kappa, \alpha}$ of the spatial operator associated to the Godunov scheme $(\kappa=1)$. This kernel is a subset of the incompressible space $\mathcal{E}_{\alpha}$. As a consequence, the Godunov scheme does not preserve any incompressible state $q \in \mathcal{E}_{\alpha}$. However, if we delete the numerical diffusion of the Godunov scheme on the velocity field by setting $\kappa=0$, the kernel of the modified equation is exactly the incompressible space $\mathcal{E}_{\alpha}$. Thus, all the incompressible states $q \in \mathcal{E}_{\alpha}$ will be preserved over time. Nevertheless, the knowledge of Ker $\mathcal{L}_{\kappa, \alpha}$ gives only partial informations on the time behaviour of the solution of (18). In the sequel, we give the definition of an accurate scheme at low Mach number when its first order modified equation is (18) and we prove that the Godunov scheme is not accurate at low Mach number when $M \ll \min (\Delta x, \Delta y)$. 


\subsection{Definition of an accurate scheme at low Mach number}

We propose the following definition in order to clearly define an accurate scheme at low Mach number:

Definition 7.1. Scheme (17) is accurate at low Mach number if the solution $q(t, \boldsymbol{x})$ of the modified equation (18) related to this scheme satisfies

$$
\begin{aligned}
\forall\left(C_{1}, C_{2}\right) \in\left(\mathbb{R}_{*}^{+}\right)^{2}, \exists C_{3}>0 \text { such that }\left\|q^{0}-\mathbb{P}_{\alpha} q^{0}\right\|_{L_{\alpha}^{2}} & =C_{1} M \\
& \Longrightarrow \forall t \in\left[0, C_{2} M\right],\left\|q-\mathbb{P}_{\alpha} q^{0}\right\|_{L_{\alpha}^{2}}(t) \leq C_{3} M .
\end{aligned}
$$

We underline that $C_{3}$ does not depend on $M$ and we recall that $\mathbb{P}_{\alpha}$ is the orthogonal projection on $\mathcal{E}_{\alpha}$ defined by (8).

This definition is justified by the fact that the solution of the linear wave equation (3) satisfies (22) (see [5] for an accurate justification of this definition).

\subsection{Inaccuracy of the Godunov scheme at low Mach number}

The following theorem - written in 2D for the sake of simplicity, the 3D case being similar - explains why the Godunov scheme applied to the linear wave equation with porosity on a cartesian mesh is not accurate at low Mach number:

Theorem 7.2. When

$$
\min (\Delta x, \Delta y) \leq \sqrt{2} \operatorname{diam}(\mathbb{T}) \sqrt{\|\alpha\|_{\infty} \cdot\left\|\frac{1}{\alpha}\right\|_{\infty}},
$$

for almost all initial conditions $q^{0} \in L_{\alpha}^{2}(\mathbb{T})^{3}$, the solution $q(t, \boldsymbol{x})$ of $(18)$ with $\kappa=1$ verifies:

$$
\begin{aligned}
\exists\left(C_{2}, C_{3}\right) \in\left(\mathbb{R}_{*}^{+}\right)^{2} \text { such that } \forall C_{1}>0,\left\|q^{0}-\mathbb{P}_{\alpha} q^{0}\right\|_{L_{\alpha}^{2}} & =C_{1} M \\
& \Longrightarrow \forall t \geq C_{2} M,\left\|q-\mathbb{P}_{\alpha} q^{0}\right\|_{L_{\alpha}^{2}}(t) \geq C_{3} \min (\Delta x, \Delta y)
\end{aligned}
$$

for any $M \leq \frac{C_{3}}{C_{1}} \min (\Delta x, \Delta y), C_{2}$ and $C_{3}$ being positive parameters that do not depend on $M, \Delta x$ and $\Delta y$.

This result - which is a generalization of Point 1 of Theorem 4.1 in [5] obtained with a constant porosity - shows that the Godunov scheme is not accurate at low Mach number when $M \ll \min (\Delta x, \Delta y)$ (for almost all initial condition $\left.q^{0}\right)$ since it does not verify (22). Let us note that (23) is verified when $\min (\Delta x, \Delta y) \leq \sqrt{2 \alpha_{\min }} \operatorname{diam}(\mathbb{T})$ because

$$
0<\alpha_{\min } \leq \alpha_{\max } \leq 1 \Longrightarrow 0<\alpha_{\min } \leq \sqrt{\alpha_{\max }} \Longrightarrow \sqrt{\alpha_{\min }} \leq \sqrt{\frac{\alpha_{\max }}{\alpha_{\min }}}=\sqrt{\|\alpha\|_{\infty} \cdot\left\|\frac{1}{\alpha}\right\|_{\infty}}
$$

and that $\min (\Delta x, \Delta y) \leq \sqrt{2 \alpha_{\min }} \operatorname{diam}(\mathbb{T})$ is easily satisfied (we underline that $\alpha_{\min }$ is of order one in the sense that $\left.M \ll \alpha_{\min }\right)$.

\subsection{Proof of Theorem 7.2}

By linearity, the solution $q(t, \mathbf{x})$ of (18) with the initial condition $q^{0}$ can be written as $q(t, \mathbf{x})=q_{1}(t, \mathbf{x})+$ $q_{2}(t, \mathbf{x})$, where $q_{1}$ is solution of

$$
\left\{\begin{array}{l}
\partial_{t}\left(\alpha q_{1}\right)+\frac{\mathcal{L}_{\kappa, \alpha}}{M}\left(q_{1}\right)=0, \\
q_{1}(t=0, \mathbf{x})=\left(q^{0}-\mathbb{P}_{\alpha} q^{0}\right)(\mathbf{x})
\end{array}\right.
$$


and $q_{2}$ is the solution of

We have

$$
\left\{\begin{array}{l}
\partial_{t}\left(\alpha q_{2}\right)+\frac{\mathcal{L}_{\kappa, \alpha}}{M}\left(q_{2}\right)=0 \\
q_{2}(t=0, \mathbf{x})=\mathbb{P}_{\alpha} q^{0}(\mathbf{x})
\end{array}\right.
$$

$$
\begin{aligned}
\forall t \geq 0, \quad\left\|q-\mathbb{P}_{\alpha} q^{0}\right\|_{L_{\alpha}^{2}}(t) & =\left\|q_{1}+q_{2}-\mathbb{P}_{\alpha} q^{0}\right\|_{L_{\alpha}^{2}}(t) \geq\left\|q_{2}-\mathbb{P}_{\alpha} q^{0}\right\|_{L_{\alpha}^{2}}(t)-\left\|q_{1}\right\|_{L_{\alpha}^{2}}(t) \\
& \geq\left\|q_{2}-\mathbb{P}_{\alpha} q^{0}\right\|_{L_{\alpha}^{2}}(t)-\left\|q_{1}\right\|_{L_{\alpha}^{2}}(0)=\left\|q_{2}-\mathbb{P}_{\alpha} q^{0}\right\|_{L_{\alpha}^{2}}(t)-\left\|q^{0}-\mathbb{P}_{\alpha} q^{0}\right\|_{L_{\alpha}^{2}}
\end{aligned}
$$

because Equation (18) is dissipative when $\kappa \geq 0$ (see Lemma 6.2). Then, if $\left\|q^{0}-\mathbb{P}_{\alpha} q^{0}\right\|_{L_{\alpha}^{2}}=C_{1} M$, we only have to study the function $t \mapsto\left\|q_{2}-\mathbb{P}_{\alpha} q^{0}\right\|_{L_{\alpha}^{2}}(t)$, where $q_{2}$ is the solution of (26). The idea is to find a lower bound for the function $t \mapsto\left\|q_{2}-\mathbb{P}_{\alpha} q^{0}\right\|_{L_{\alpha}^{2}}(t)$. To do this, we need some tools:

- a projection $\mathbb{P}_{\kappa=1, \alpha}$ on $\operatorname{Ker} \mathcal{L}_{\kappa=1, \alpha}$ where $\operatorname{Ker} \mathbb{P}_{\kappa=1, \alpha}$ is invariant for Equation (18) (in the sense of (28)),

- we write

$$
q_{2}-\mathbb{P}_{\alpha} q^{0}=q_{2}-\mathbb{P}_{\kappa=1, \alpha} \mathbb{P}_{\alpha} q^{0}+\mathbb{P}_{\kappa=1, \alpha} \mathbb{P}_{\alpha} q^{0}-\mathbb{P}_{\alpha} q^{0},
$$

- we verify that $\mathbb{P}_{\kappa=1, \alpha}\left(q_{2}-\mathbb{P}_{\kappa=1, \alpha} \mathbb{P}_{\alpha} q^{0}\right)=0$,

- we use a Poincaré-Wirtinger inequality valid on $\operatorname{Ker} \mathbb{P}_{\kappa=1, \alpha}$ for $q_{2}-\mathbb{P}_{\kappa=1, \alpha} \mathbb{P}_{\alpha} q^{0}$,

- we verify that $q_{2}-\mathbb{P}_{\kappa=1, \alpha} \mathbb{P}_{\alpha} q^{0}$ is solution of (18),

- we obtain the rate of dissipation of $\left\|q_{2}-\mathbb{P}_{\kappa=1, \alpha} \mathbb{P}_{\alpha} q^{0}\right\|$ to zero by applying the Grönwall's lemma.

Lemma 7.3. The function

$$
\begin{aligned}
& L_{\alpha}^{2}(\mathbb{T})^{3} \rightarrow \operatorname{Ker} \mathcal{L}_{\kappa=1, \alpha} \\
& q=\left(\begin{array}{c}
r \\
\boldsymbol{u}
\end{array}\right) \mapsto\left(\begin{array}{c}
\frac{1}{\int_{\mathbb{T}} \alpha d x d y} \int_{\mathbb{T}} r \alpha d x d y \\
\frac{1}{\alpha \int_{a_{1}}^{b_{1}} \frac{1}{\alpha(x, y)} d x} \int_{a_{1}}^{b_{1}} u_{x}(x, y) d x \\
\frac{1}{\alpha \int_{a_{2}}^{b_{2}} \frac{1}{\alpha(x, y)} d y} \int_{a_{2}}^{b_{2}} u_{y}(x, y) d y
\end{array}\right)
\end{aligned}
$$

defines a projection $\mathbb{P}_{\kappa=1, \alpha}$. Moreover, if $q(t, \boldsymbol{x})$ is the solution of (18) on $\mathbb{T}$ with initial condition $q^{0}$ :

$$
\forall q^{0} \in \operatorname{Ker} \mathbb{P}_{\kappa=1, \alpha}: \quad q(t \geq 0) \in \operatorname{Ker} \mathbb{P}_{\kappa=1, \alpha} .
$$

Proof. Recall that $\mathbb{T}=\left[a_{1}, b_{1}\right] \times\left[a_{2}, b_{2}\right]$. It is easy to prove that $\mathbb{P}_{\kappa=1, \alpha} \circ \mathbb{P}_{\kappa=1, \alpha}=\mathbb{P}_{\kappa=1, \alpha}$. This proves that $\mathbb{P}_{\kappa=1, \alpha}$ is a projector. Moreover, we have $\operatorname{Im} \mathbb{P}_{\kappa=1, \alpha} \subset \operatorname{Ker} \mathcal{L}_{\kappa=1, \alpha}$ because for all $q=(r, \mathbf{u})^{T} \in L_{\alpha}^{2}(\mathbb{T})^{1+d}$,

$$
\begin{aligned}
& \nabla\left(\frac{1}{\int_{\mathbb{T}} \alpha d x d y} \int_{\mathbb{T}} r \alpha d x d y\right)=0 \\
& \partial_{x}\left(\alpha \frac{1}{\alpha \int_{a_{1}}^{b_{1}} \frac{1}{\alpha(x, y)} d x} \int_{a_{1}}^{b_{1}} u_{x}(x, y) d x\right)=\partial_{x}\left(\frac{1}{\int_{a_{1}}^{b_{1}} \frac{1}{\alpha(x, y)} d x} \int_{a_{1}}^{b_{1}} u_{x}(x, y) d x\right)=0 \\
& \partial_{y}\left(\alpha \frac{1}{\alpha \int_{a_{2}}^{b_{2}} \frac{1}{\alpha(x, y)} d y} \int_{a_{2}}^{b_{2}} u_{y}(x, y) d y\right)=\partial_{y}\left(\frac{1}{\int_{a_{2}}^{b_{2}} \frac{1}{\alpha(x, y)} d y} \int_{a_{2}}^{b_{2}} u_{y}(x, y) d y\right)=0
\end{aligned}
$$


Let $q(t, \mathbf{x})$ be a solution of (18) on $\mathbb{T}$ with initial condition $q^{0} \in \operatorname{Ker} \mathbb{P}_{\kappa=1, \alpha}$. By integrating the first equation of system $(18)$ on $\mathbb{T}$, we obtain

$$
\frac{d}{d t} \int_{\mathbb{T}} r \alpha d x d y+\frac{a_{\star}}{M} \int_{\mathbb{T}} \nabla \cdot(\alpha \mathbf{u}) d x d y=\int_{\mathbb{T}}\left(\frac{a_{\star} \Delta x}{2 M} \partial_{x}\left(\alpha \partial_{x} r\right)+\frac{a_{\star} \Delta y}{2 M} \partial_{y}\left(\alpha \partial_{y} r\right)\right) d x d y \Rightarrow \frac{d}{d t} \int_{\mathbb{T}} \alpha r d x d y=0
$$

by periodicity. Then, if $\int_{\mathbb{T}} \alpha r^{0} d x d y=0$, it is the case at any time. Moreover, since $\alpha$ does not depend on time, we can write the second equation of (18) under the form

$$
\partial_{t} u_{x}+\frac{a_{\star}}{M} \partial_{x} r=\kappa \frac{a_{\star} \Delta x}{2 M} \partial_{x}\left(\frac{1}{\alpha} \partial_{x}\left(\alpha u_{x}\right)\right) .
$$

By integrating on $\left[a_{1}, b_{1}\right]$, we obtain

$$
\partial_{t} \int_{a_{1}}^{b_{1}} u_{x}(x, y) d x+\frac{a_{\star}}{M} \int_{a_{1}}^{b_{1}} \partial_{x} r d x=\kappa \frac{a_{\star} \Delta x}{2 M} \int_{a_{1}}^{b_{1}} \partial_{x}\left(\frac{1}{\alpha} \partial_{x}\left(\alpha u_{x}\right)\right) d x \Rightarrow \partial_{t} \int_{a_{1}}^{b_{1}} u_{x}(x, y) d x=0
$$

by periodicity. Then, if $\int_{a_{1}}^{b_{1}} u_{x}^{0}(x, y) d x=0$, it is the case at any time. We apply the same technique for $u_{y}$ and we obtain that (28) is satisfied.

We now write a Poincaré-Wirtinger inequality for a function $q \in \operatorname{Ker} \mathbb{P}_{\kappa=1, \alpha}$ :

Lemma 7.4. For any $q:=\left(r, u_{x}, u_{y}\right)^{T} \in \operatorname{Ker} \mathbb{P}_{\kappa=1, \alpha}$ such that $\left(r, \alpha u_{x}, \alpha u_{y}\right)^{T} \in H_{1}(\mathbb{T})^{3}$, we have

$$
\|q\|_{L_{\alpha}^{2}}^{2} \leq K_{\alpha}(\mathbb{T})^{2}\left(\|\nabla r\|_{L_{\alpha}^{2}}^{2}+\left\|\frac{\partial_{x}\left(\alpha u_{x}\right)}{\alpha}\right\|_{L_{\alpha}^{2}}^{2}+\left\|\frac{\partial_{y}\left(\alpha u_{y}\right)}{\alpha}\right\|_{L_{\alpha}^{2}}^{2}\right)
$$

with $K_{\alpha}(\mathbb{T})=\sqrt{2} \operatorname{diam}(\mathbb{T}) \sqrt{\|\alpha\|_{\infty} \cdot\left\|\frac{1}{\alpha}\right\|_{\infty}}$.

Proof. Let $q=\left(r, u_{x}, u_{y}\right)^{T} \in \operatorname{Ker} \mathbb{P}_{\kappa=1, \alpha}$. Since $\int_{\mathbb{T}} r \alpha d x d y=0$, by using the weighted Poincaré-Wirtinger inequality (11) on $r$, we obtain

$$
\|r\|_{L_{\alpha}^{2}} \leq \frac{1}{\sqrt{C_{\alpha}(\mathbb{T})}}\|\nabla r\|_{L_{\alpha}^{2}}
$$

where $\sqrt{C_{\alpha}(\mathbb{T})}=1 / K_{\alpha}(\mathbb{T})$. Moreover, since for all $y \in\left[a_{2}, b_{2}\right]$, we have $0=\int_{a_{1}}^{b_{1}} u_{x}(x, y) d x=\int_{a_{1}}^{b_{1}}\left(\alpha u_{x}\right)(x, y) \frac{1}{\alpha} d x$, by applying the weighted Poincaré-Wirtinger inequality (10) to the function $x \mapsto\left(\alpha u_{x}\right)(x, y)$ with the weight $\mu=\frac{1}{\alpha}$, we obtain

$$
\int_{a_{1}}^{b_{1}}\left|\left(\alpha u_{x}\right)(x, y)\right|^{2} \frac{1}{\alpha} d x \leq \frac{1}{C_{\alpha}(\mathbb{T})} \int_{a_{1}}^{b_{1}}\left|\partial_{x}\left(\alpha u_{x}\right)(x, y)\right|^{2} \frac{1}{\alpha} d x
$$

that is to say

$$
\int_{a_{1}}^{b_{1}}\left|u_{x}(x, y)\right|^{2} \alpha d x \leq \frac{1}{C_{\alpha}(\mathbb{T})} \int_{a_{1}}^{b_{1}}\left|\frac{\partial_{x}\left(\alpha u_{x}\right)(x, y)}{\alpha}\right|^{2} \alpha d x
$$

Thus, by integrating over $\left[a_{2}, b_{2}\right]$, we find

$$
\left\|u_{x}\right\|_{L_{\alpha}^{2}}^{2}(t) \leq \frac{1}{C_{\alpha}(\mathbb{T})}\left\|\frac{\partial_{x}\left(\alpha u_{x}\right)}{\alpha}\right\|_{L_{\alpha}^{2}}^{2}(t)
$$


We apply the same analysis for $u_{y}$ such that $0=\int_{a_{2}}^{b_{2}} u_{y}(x, y) d y=\int_{a_{2}}^{b_{2}}\left(\alpha u_{y}\right)(x, y) \frac{1}{\alpha} d y$, which gives

$$
\left\|u_{y}\right\|_{L_{\alpha}^{2}}^{2}(t) \leq \frac{1}{C_{\alpha}(\mathbb{T})}\left\|\frac{\partial_{y}\left(\alpha u_{y}\right)}{\alpha}\right\|_{L_{\alpha}^{2}}^{2}(t) .
$$

We obtain (29) with (30), (31) and (32).

To prove inequality (24), we firstly have to prove the following lemma:

Lemma 7.5. There exists a constant $K_{\alpha}(\mathbb{T})>0$ depending on $\mathbb{T}$ and $\alpha$ such that

$$
\forall t \geq 0, \quad\left\|q_{2}-\mathbb{P}_{\kappa=1, \alpha} \mathbb{P}_{\alpha} q^{0}\right\|_{L_{\alpha}^{2}}(t) \leq\left\|\left(1-\mathbb{P}_{\kappa=1, \alpha}\right) \circ \mathbb{P}_{\alpha} q^{0}\right\|_{L_{\alpha}^{2}} \exp \left(-\frac{a_{\star} \min (\Delta x, \Delta y)}{2 M K_{\alpha}(\mathbb{T})^{2}} t\right) .
$$

Proof. Let us define $\hat{q}=q_{2}-\mathbb{P}_{\kappa=1, \alpha} \mathbb{P}_{\alpha} q^{0}=:(\hat{r}, \hat{\mathbf{u}})^{T}$. The idea is to apply the inequality of Lemma 7.4 combined with the equality (21) in the proof of Lemma 6.2. For this, we firstly prove that $\hat{q}$ satisfies (18). Since $q_{2}$ satisfies (18), $\hat{q}$ satisfies (18) if and only if $\mathbb{P}_{\kappa=1, \alpha} \mathbb{P}_{\alpha} q^{0}$ satisfies (18). Since $\operatorname{Im} \mathbb{P}_{\kappa=1, \alpha} \subset \operatorname{Ker} \mathcal{L}_{\kappa=1, \alpha}$, we have $\mathcal{L}_{\kappa=1, \alpha}\left(\mathbb{P}_{\kappa=1, \alpha} \mathbb{P}_{\alpha} q^{0}\right)=0$ and then $\mathbb{P}_{\kappa=1, \alpha} \mathbb{P}_{\alpha} q^{0}$ satisfies (18). Then, $\hat{q}$ is solution of (18) and by using (21), we obtain

$$
\begin{aligned}
\frac{1}{2} \frac{d}{d t}\|\hat{q}\|_{L_{\alpha}^{2}}^{2}(t) & =-\frac{a_{\star}}{2 M}\left(\Delta x\left\|\partial_{x} \hat{r}\right\|_{L_{\alpha}^{2}}^{2}+\Delta y\left\|\partial_{y} \hat{r}\right\|_{L_{\alpha}^{2}}^{2}(t)+\kappa \Delta x\left\|\frac{\partial_{x}\left(\alpha \hat{u}_{x}\right)}{\alpha}\right\|_{L_{\alpha}^{2}}^{2}(t)+\kappa \Delta y\left\|\frac{\partial_{y}\left(\alpha \hat{u}_{y}\right)}{\alpha}\right\|_{L_{\alpha}^{2}}^{2}(t)\right) \\
& \leq-\frac{a_{\star}}{2 M} \min (\Delta x, \Delta y)\left(\|\nabla \hat{r}\|_{L_{\alpha}^{2}}^{2}(t)+\left\|\frac{\partial_{x}\left(\alpha \hat{u}_{x}\right)}{\alpha}\right\|_{L_{\alpha}^{2}}^{2}(t)+\left\|\frac{\partial_{y}\left(\alpha \hat{u}_{y}\right)}{\alpha}\right\|_{L_{\alpha}^{2}}^{2}(t)\right)
\end{aligned}
$$

since $\kappa=1$. Since $\mathbb{P}_{\kappa=1, \alpha} \hat{q}(t=0)=\mathbb{P}_{\kappa=1, \alpha}\left(q_{2}^{0}-\mathbb{P}_{\kappa=1, \alpha} \mathbb{P}_{\alpha} q^{0}\right)=\mathbb{P}_{\kappa=1, \alpha} \circ \mathbb{P}_{\alpha} q^{0}-\mathbb{P}_{\kappa=1, \alpha} \circ \mathbb{P}_{\alpha} q^{0}=0$ and since Ker $\mathbb{P}_{\kappa=1, \alpha}$ is invariant for Equation (18) (in the sense of (28)), we have $\hat{q}(t \geq 0) \in \operatorname{Ker} \mathbb{P}_{\kappa=1, \alpha}$. Thus, we can apply Lemma 7.4 to $\hat{q}$ and we obtain from (34)

$$
\frac{1}{2} \frac{d}{d t}\|\hat{q}\|_{L_{\alpha}^{2}}^{2}(t) \leq-\frac{a_{\star} \min (\Delta x, \Delta y)}{2 M K_{\alpha}(\mathbb{T})^{2}}\|\hat{q}\|_{L_{\alpha}^{2}}^{2}(t) .
$$

By applying the Grönwall's lemma, we obtain (33) because $\hat{q}(t=0)=\left(1-\mathbb{P}_{\kappa=1, \alpha}\right) \circ \mathbb{P}_{\alpha} q^{0}$.

Now, we are able to prove Theorem 7.2. By applying Lemma 7.5, we have for all $t \geq 0$ :

$$
\begin{aligned}
\left\|q_{2}-\mathbb{P}_{\alpha} q^{0}\right\|_{L_{\alpha}^{2}}(t) & \geq\left\|\mathbb{P}_{\alpha} q^{0}-\mathbb{P}_{\kappa=1, \alpha} \mathbb{P}_{\alpha} q^{0}\right\|_{L_{\alpha}^{2}}-\left\|q_{2}-\mathbb{P}_{\kappa=1, \alpha} \mathbb{P}_{\alpha} q^{0}\right\|_{L_{\alpha}^{2}}(t) \\
& \geq\left\|\left(1-\mathbb{P}_{\kappa=1, \alpha}\right) \circ \mathbb{P}_{\alpha} q^{0}\right\|_{L_{\alpha}^{2}}\left(1-\exp \left(-\frac{a_{\star} \min (\Delta x, \Delta y)}{2 M K_{\alpha}(\mathbb{T})^{2}} t\right)\right) .
\end{aligned}
$$

By noting that $1-\exp (-x / 2) \geq x / 3$ for $x \in[0,1]$, we have

$$
\forall t \leq \frac{M K_{\alpha}(\mathbb{T})^{2}}{a_{\star} \min (\Delta x, \Delta y)}: \quad 1-\exp \left(-\frac{a_{\star} \min (\Delta x, \Delta y)}{2 M K_{\alpha}(\mathbb{T})^{2}} t\right) \geq \frac{a_{\star} \min (\Delta x, \Delta y)}{3 M K_{\alpha}(\mathbb{T})^{2}} t .
$$

Thus, we have also

$$
\forall t \in\left[\frac{M K_{\alpha}(\mathbb{T})}{a_{\star}}, \frac{M K_{\alpha}(\mathbb{T})^{2}}{a_{\star} \min (\Delta x, \Delta y)}\right]: \quad 1-\exp \left(-\frac{a_{\star} \min (\Delta x, \Delta y)}{2 M K_{\alpha}(\mathbb{T})^{2}} t\right) \geq \frac{\min (\Delta x, \Delta y)}{3 K_{\alpha}(\mathbb{T})}
$$


when $\min (\Delta x, \Delta y) \leq K_{\alpha}(\mathbb{T})$. Moreover, we have

$$
\forall t \geq \frac{M K_{\alpha}(\mathbb{T})^{2}}{a_{\star} \min (\Delta x, \Delta y)}: \quad 1-\exp \left(-\frac{a_{\star} \min (\Delta x, \Delta y)}{2 M K_{\alpha}(\mathbb{T})^{2}} t\right) \geq 1-\frac{1}{\sqrt{e}}
$$

Then, if $\min (\Delta x, \Delta y) \leq K_{\alpha}(\mathbb{T})$, we have

$$
\forall t \geq \frac{M K_{\alpha}(\mathbb{T})}{a_{\star}}: \quad 1-\exp \left(-\frac{a_{\star} \min (\Delta x, \Delta y)}{2 M K_{\alpha}(\mathbb{T})^{2}} t\right) \geq \min \left(\frac{\min (\Delta x, \Delta y)}{3 K_{\alpha}(\mathbb{T})}, 1-\frac{1}{\sqrt{e}}\right)=\frac{\min (\Delta x, \Delta y)}{3 K_{\alpha}(\mathbb{T})}
$$

because $\frac{1}{3} \leq 1-\frac{1}{\sqrt{e}}$. Then, if $\min (\Delta x, \Delta y) \leq K_{\alpha}(\mathbb{T})$, we have

$$
\begin{aligned}
\forall t \geq C_{2} M: \quad\left\|q_{2}-\mathbb{P}_{\alpha} q^{0}\right\|_{L_{\alpha}^{2}}(t) & \geq\left\|\left(1-\mathbb{P}_{\kappa=1, \alpha}\right) \circ \mathbb{P}_{\alpha} q^{0}\right\|_{L_{\alpha}^{2}} \frac{\min (\Delta x, \Delta y)}{3 K_{\alpha}(\mathbb{T})} \\
& \geq C \min (\Delta x, \Delta y)
\end{aligned}
$$

with $C_{2}=\frac{K_{\alpha}(\mathbb{T})}{a_{\star}}$ and $C=\frac{\left\|\left(1-\mathbb{P}_{\kappa=1, \alpha}\right) \circ \mathbb{P}_{\alpha} q^{0}\right\|_{L_{\alpha}^{2}}}{3 K_{\alpha}(\mathbb{T})}$. In the sequel, we suppose that $C$ is strictly positive, which is the case for all function $q^{0} \in L_{\alpha}^{2}(\mathbb{T})^{3}$ such that $\mathbb{P}_{\alpha} q^{0} \notin \operatorname{Ker} \mathcal{L}_{\kappa=1, \alpha}$. Moreover, since Equation (18) is dissipative when $\kappa \geq 0$ (see Lemma 6.2), we can write that

$$
C_{1} M=\left\|q_{1}\right\|_{L_{\alpha}^{2}}(0) \geq\left\|q_{1}\right\|_{L_{\alpha}^{2}}(t) .
$$

Let us now suppose that $C_{1} M \leq C \min (\Delta x, \Delta y)$. Then, by using (27) and (35), we find

$$
\forall t \geq C_{2} M: \quad\left\|q-\mathbb{P}_{\alpha} q^{0}\right\|_{L_{\alpha}^{2}}(t) \geq\left\|q_{2}-\mathbb{P}_{\alpha} q^{0}\right\|_{L_{\alpha}^{2}}(t)-\left\|q_{1}\right\|_{L_{\alpha}^{2}}(t) \geq C \min (\Delta x, \Delta y)-C_{1} M \geq 0 .
$$

Let us now suppose that $C_{1} M \leq C_{3} \min (\Delta x, \Delta y)$ with $C_{3}=\frac{C}{2}$. This gives

$$
\forall t \geq C_{2} M: \quad\left\|q-\mathbb{P}_{\alpha} q^{0}\right\|_{L_{\alpha}^{2}}(t) \geq C_{3} \min (\Delta x, \Delta y)
$$

for any $M \leq \frac{C_{3}}{C_{1}} \min (\Delta x, \Delta y)$. This concludes the proof of Theorem 7.2.

\section{Low Mach and all Mach Corrections for the Godunov scheme to Be ACCURATE AT LOW MACH NUMBER ON A CARTESIAN MESH}

Theorem 7.2 shows that the Godunov scheme is not accurate (in the sense of Definition 7.1) at low Mach number when $M \ll \min (\Delta x, \Delta y)$. We now prove that the Godunov scheme is accurate when $\max (\Delta x, \Delta y)$ is of the order of $M$. Of course, this condition on the mesh is too expensive for practical applications. To overcome this difficulty, we propose a low Mach correction which allows to recover the accuracy when $M \ll \min (\Delta x, \Delta y)$. At last, we propose an all Mach correction which allows to recover the accuracy at low Mach number when $M \ll \min (\Delta x, \Delta y)$ and the Godunov scheme when the Mach number is of order one (when the porosity is constant, we show in [5] that this all Mach correction may be more robust than the low Mach correction when it is applied in the linear case with linear convection and in the non-linear case (1)). These three points are detailed in the following theorem:

Theorem 8.1. Let $q(t, \boldsymbol{x})$ be the solution of (18) with the initial condition $q^{0}$. We have: 
1) The Godunov scheme - obtained with $\kappa=1$ in (17) - is accurate at low Mach number when

$$
\max (\Delta x, \Delta y)=\mathcal{O}(M)
$$

More precisely:

$$
\begin{aligned}
\forall\left(C_{0}, C_{1}, C_{2}\right) \in\left(\mathbb{R}_{*}^{+}\right)^{3}, \exists C_{3}>0 \text { such that }\left\{\begin{array}{l}
\Delta x \leq C_{0} M, \\
\Delta y \leq C_{0} M, \\
\left\|q^{0}-\mathbb{P}_{\alpha} q^{0}\right\|_{L_{\alpha}^{2}}=C_{1} M \\
\end{array} \quad \forall t \in\left[0, C_{2} M\right],\left\|q-\mathbb{P}_{\alpha} q^{0}\right\|_{L_{\alpha}^{2}}(t) \leq C_{3} M\right.
\end{aligned}
$$

where $C_{3}$ does not depend on $M, \Delta x$ and $\Delta y$.

2) The low Mach Godunov scheme - obtained with $\kappa=0$ in (17) - is accurate at low Mach number. More precisely:

$$
\forall C_{1} \in \mathbb{R}_{*}^{+}, \quad\left\|q^{0}-\mathbb{P}_{\alpha} q^{0}\right\|_{L_{\alpha}^{2}}=C_{1} M \quad \Longrightarrow \quad \forall t \geq 0,\left\|q-\mathbb{P}_{\alpha} q^{0}\right\|_{L_{\alpha}^{2}}(t) \leq C_{1} M
$$

3) The all Mach Godunov scheme - obtained with $\kappa=\min (1, M)$ in $(17)$ - is accurate at low Mach number. More precisely:

$$
\begin{aligned}
\forall\left(C_{1}, C_{2}\right) \in\left(\mathbb{R}_{*}^{+}\right)^{2}, \exists C_{3}>0 \text { such that }\left\|q^{0}-\mathbb{P}_{\alpha} q^{0}\right\|_{L_{\alpha}^{2}}=C_{1} M & \\
& \Longrightarrow \forall t \in\left[0, C_{2} M\right],\left\|q-\mathbb{P}_{\alpha} q^{0}\right\|_{L_{\alpha}^{2}}(t) \leq C_{3} M
\end{aligned}
$$

where $C_{3}$ does not depend on $M$.

Proof. By linearity, the solution $q(t, \mathbf{x})$ of $(18)$ with the initial condition $q^{0}$ can be written as

$$
q(t, \mathbf{x})=q_{1}(t, \mathbf{x})+q_{2}(t, \mathbf{x})
$$

where $q_{1}$ is the solution of (25) and $q_{2}$ is the solution of (26). We have

$$
\begin{aligned}
\forall t \geq 0, \quad\left\|q-\mathbb{P}_{\alpha} q^{0}\right\|_{L_{\alpha}^{2}}(t) & =\left\|q_{1}+q_{2}-\mathbb{P}_{\alpha} q^{0}\right\|_{L_{\alpha}^{2}}(t) \leq\left\|q_{1}\right\|_{L_{\alpha}^{2}}(t)+\left\|q_{2}-\mathbb{P}_{\alpha} q^{0}\right\|_{L_{\alpha}^{2}}(t) \\
& \leq\left\|q^{0}-\mathbb{P}_{\alpha} q^{0}\right\|_{L_{\alpha}^{2}}+\left\|q_{2}-\mathbb{P}_{\alpha} q^{0}\right\|_{L_{\alpha}^{2}}(t)
\end{aligned}
$$

because Equation (18) is dissipative when $\kappa \geq 0$ (see Lemma 6.2). Then, if $\left\|q^{0}-\mathbb{P}_{\alpha} q^{0}\right\|_{L_{\alpha}^{2}}=C_{1} M$, we just have to study the function $t \mapsto\left\|q_{2}-\mathbb{P}_{\alpha} q^{0}\right\|_{L_{\alpha}^{2}}(t)$, where $q_{2}$ is the solution of (26). Since $\mathbb{P}_{\alpha} q^{0} \in \mathcal{E}_{\alpha}=\operatorname{Ker} L_{\alpha}$, we have $L_{\alpha}\left(\mathbb{P}_{\alpha} q^{0}\right)=0$ and

which implies, by using (26), that

$$
\partial_{t}\left(\alpha \mathbb{P}_{\alpha} q^{0}\right)+\frac{L_{\alpha}}{M}\left(\mathbb{P}_{\alpha} q^{0}\right)=0
$$

$$
\partial_{t}\left(\alpha\left(q_{2}-\mathbb{P}_{\alpha} q^{0}\right)\right)+\frac{L_{\alpha}}{M}\left(q_{2}-\mathbb{P}_{\alpha} q^{0}\right)=B_{\kappa, \alpha}\left(q_{2}-\mathbb{P}_{\alpha} q^{0}\right)+B_{\kappa, \alpha}\left(\mathbb{P}_{\alpha} q^{0}\right)
$$


By multiplying (40) with $\left(q_{2}-\mathbb{P}_{\alpha} q^{0}\right)$, by integrating over $\mathbb{T}$ and by using (13), we obtain

$$
\left\langle\frac{q_{2}-\mathbb{P}_{\alpha} q^{0}}{\alpha}, \partial_{t}\left(\alpha\left(q_{2}-\mathbb{P}_{\alpha} q^{0}\right)\right)\right\rangle_{\alpha}+0=\left\langle\frac{q_{2}-\mathbb{P}_{\alpha} q^{0}}{\alpha}, B_{\kappa, \alpha}\left(q_{2}-\mathbb{P}_{\alpha} q^{0}\right)\right\rangle_{\alpha}+\left\langle\frac{q_{2}-\mathbb{P}_{\alpha} q^{0}}{\alpha}, B_{\kappa, \alpha}\left(\mathbb{P}_{\alpha} q^{0}\right)\right\rangle_{\alpha}
$$

Since (20) allows to write that $\left\langle\frac{q_{2}-\mathbb{P}_{\alpha} q^{0}}{\alpha}, B_{\kappa, \alpha}\left(q_{2}-\mathbb{P}_{\alpha} q^{0}\right)\right\rangle_{\alpha} \leq 0$, we obtain

$$
\frac{1}{2} \frac{d}{d t}\left\|q_{2}-\mathbb{P}_{\alpha} q^{0}\right\|_{L_{\alpha}^{2}}^{2}(t) \leq\left\langle\frac{q_{2}-\mathbb{P}_{\alpha} q^{0}}{\alpha}, B_{\kappa, \alpha}\left(\mathbb{P}_{\alpha} q^{0}\right)\right\rangle_{\alpha} \leq\left\|\frac{B_{\kappa, \alpha}\left(\mathbb{P}_{\alpha} q^{0}\right)}{\alpha}\right\|_{L_{\alpha}^{2}} \cdot\left\|q_{2}-\mathbb{P}_{\alpha} q^{0}\right\|_{L_{\alpha}^{2}}(t)
$$

that is to say

$$
\frac{d}{d t}\left\|q_{2}-\mathbb{P}_{\alpha} q^{0}\right\|_{L_{\alpha}^{2}}(t) \leq\left\|\frac{B_{\kappa, \alpha}\left(\mathbb{P}_{\alpha} q^{0}\right)}{\alpha}\right\|_{L_{\alpha}^{2}} .
$$

Since $\nabla\left(\mathbb{P}_{\alpha} r^{0}\right)=0$, we deduce from (20) that

$$
\left\|\frac{B_{\kappa, \alpha}\left(\mathbb{P}_{\alpha} q^{0}\right)}{\alpha}\right\|_{L_{\alpha}^{2}} \leq \max \left(\left|\kappa \frac{a_{\star} \Delta x}{2 M}\right|,\left|\kappa \frac{a_{\star} \Delta y}{2 M}\right|\right) \cdot\left(\left\|\partial_{x}\left(\frac{\partial_{x}\left(\alpha \mathbb{P}_{\alpha} u_{x}^{0}\right)}{\alpha}\right)\right\|_{L_{\alpha}^{2}}+\left\|\partial_{y}\left(\frac{\partial_{y}\left(\alpha \mathbb{P}_{\alpha} u_{y}^{0}\right)}{\alpha}\right)\right\|_{L_{\alpha}^{2}}\right) .
$$

Thus, by using the fact that $\left\|q_{2}-\mathbb{P}_{\alpha} q^{0}\right\|_{L_{\alpha}^{2}}(0)=0$, we obtain by using (41)

$$
\forall t \in\left[0, C_{2} M\right], \quad\left\|q_{2}-\mathbb{P}_{\alpha} q^{0}\right\|_{L_{\alpha}^{2}}(t) \leq C_{2} M \max \left(\left|\kappa \frac{a_{\star} \Delta x}{2 M}\right|,\left|\kappa \frac{a_{\star} \Delta y}{2 M}\right|\right) \mathcal{C}_{\alpha}\left(\mathbb{P}_{\alpha} q^{0}\right)
$$

where $\mathcal{C}_{\alpha}\left(\mathbb{P}_{\alpha} q^{0}\right):=\left(\left\|\partial_{x}\left(\frac{\partial_{x}\left(\alpha \mathbb{P}_{\alpha} u_{x}^{0}\right)}{\alpha}\right)\right\|_{L_{\alpha}^{2}}+\left\|\partial_{y}\left(\frac{\partial_{y}\left(\alpha \mathbb{P}_{\alpha} u_{y}^{0}\right)}{\alpha}\right)\right\|_{L_{\alpha}^{2}}\right)$. Thus, when $\left\|q^{0}-\mathbb{P}_{\alpha} q^{0}\right\|_{L_{\alpha}^{2}}=C_{1} M$, by using (39), we obtain

$$
\forall t \in\left[0, C_{2} M\right], \quad\left\|q-\mathbb{P}_{\alpha} q^{0}\right\|_{L_{\alpha}^{2}}(t) \leq M\left(C_{1}+C_{2} \kappa \frac{a_{\star}}{2 M} \max (\Delta x, \Delta y) \mathcal{C}_{\alpha}\left(\mathbb{P}_{\alpha} q^{0}\right)\right) .
$$

Let us now suppose that $\kappa=1$. In that case, Inequality (42) becomes

$$
\forall t \in\left[0, C_{2} M\right], \quad\left\|q-\mathbb{P}_{\alpha} q^{0}\right\|_{L_{\alpha}^{2}}(t) \leq M\left(C_{1}+C_{2} \mathcal{C}_{\alpha}\left(\mathbb{P}_{\alpha} q^{0}\right) \frac{a_{\star}}{2 M} \max (\Delta x, \Delta y)\right)
$$

which allows to obtain (36) with $C_{3}=C_{1}+C_{2} \mathcal{C}_{\alpha}\left(\mathbb{P}_{\alpha} q^{0}\right) \frac{a_{\star}}{2} C_{0}$ when $\Delta x \leq C_{0} M$ and $\Delta y \leq C_{0} M$. We now assume that $\kappa=M$. In this case, (42) can be written as

$$
\forall t \in\left[0, C_{2} M\right], \quad\left\|q-\mathbb{P}_{\alpha} q^{0}\right\|_{L_{\alpha}^{2}}(t) \leq M\left(C_{1}+C_{2} \mathcal{C}_{\alpha}\left(\mathbb{P}_{\alpha} q^{0}\right) \frac{a_{\star}}{2} \max (\Delta x, \Delta y)\right)
$$

which allows to obtain (38) with $C_{3}=C_{1}+C_{2} \mathcal{C}_{\alpha}\left(\mathbb{P}_{\alpha} q^{0}\right) \frac{a_{\star}}{2} \max (\Delta x, \Delta y)$. When $\kappa=0$, we have

$$
\left\|\frac{B_{\kappa=0, \alpha}\left(\mathbb{P}_{\alpha} q^{0}\right)}{\alpha}\right\|_{L_{\alpha}^{2}}=0 .
$$


Then, we deduce from (41) that

$$
\frac{d}{d t}\left\|q_{2}-\mathbb{P}_{\alpha} q^{0}\right\|_{L_{\alpha}^{2}}(t)=0
$$

which implies that $\left\|q_{2}-\mathbb{P}_{\alpha} q^{0}\right\|_{L_{\alpha}^{2}}(t)=0$ for any non-negative time since $\left\|q_{2}-\mathbb{P}_{\alpha} q^{0}\right\|_{L_{\alpha}^{2}}(0)=0$. As a consequence, we deduce from (39) that

$$
\forall t \geq 0, \quad\left\|q-\mathbb{P}_{\alpha} q^{0}\right\|_{L_{\alpha}^{2}}(t) \leq\left\|q^{0}-\mathbb{P}_{\alpha} q^{0}\right\|_{L_{\alpha}^{2}}
$$

which gives (37) since $\left\|q^{0}-\mathbb{P}_{\alpha} q^{0}\right\|_{L_{\alpha}^{2}}=C_{1} M$.

\section{Numerical RESUlts}

We illustrate Theorem 7.2 and Theorem 8.1 with an initial condition $q^{0}$. We choose an initial condition $q^{0}$ such that $q^{0}=M q_{1}^{0}+q_{2}^{0}$ where $q_{1}^{0} \in \mathcal{E}_{\alpha}^{\perp},\left\|q_{1}^{0}\right\|_{L_{\alpha}^{2}}=1$ and $q_{2}^{0} \in \mathcal{E}_{\alpha}$. The function $q_{1}^{0}:=\left(r_{1}^{0} \text {, } \mathbf{u}_{1}^{0}\right)^{T}$ is given by $q_{1}^{0}=\frac{\bar{q}_{1}}{\left\|\bar{q}_{1}\right\|_{L_{\alpha}^{2}}}$ with

$$
\left\{\begin{array} { l } 
{ \overline { r } _ { 1 } ^ { 0 } ( x , y ) = \frac { \operatorname { s i n } ( 2 \pi x ) \operatorname { c o s } ( 2 \pi y ) } { \alpha ( x , y ) } , } \\
{ \overline { \mathbf { u } } _ { 1 } ^ { 0 } = \nabla \phi }
\end{array} \quad \text { where } \quad \left\{\begin{array}{l}
\alpha(x, y)=\frac{1}{2}+\frac{1}{4} \sin (\pi x) \sin (2 \pi y) \\
\phi(x, y)=\sin (2 \pi x) \cos (2 \pi y) .
\end{array}\right.\right.
$$

The functions $\alpha, r_{1}$ and $\phi$ are defined at the cell center. The function $q_{2}^{0}:=\left(r_{2}^{0}, \mathbf{u}_{2}^{0}\right)^{T}$ is given by

$$
\left\{\begin{array}{l}
r_{2}^{0}=1 \\
\mathbf{u}_{2}^{0}=\frac{\nabla \times \psi}{\alpha},
\end{array} \quad \text { where } \quad \psi(x, y)=\frac{1}{\pi} \sin ^{2}(\pi x) \sin ^{2}(\pi y)\right.
$$

By construction, we have $q_{1}^{0} \in \mathcal{E}_{\alpha}^{\perp}$ with $\left\|q_{1}^{0}\right\|_{L_{\alpha}^{2}}=1$ and $q_{2} \in \mathcal{E}_{\alpha}$. Moreover, we choose the parameters $a_{\star}=1$ and $M=10^{-2}$. Concerning the time discretization, we use an explicit scheme with a time step $\Delta t$ based on the acoustic CFL condition and given by $\Delta t=\mathrm{CFL} \times M \frac{\min (\Delta x, \Delta y)}{a_{\star}}$ with $\mathrm{CFL}=0.4$ (we recall that $a_{\star} / M$ is the sound speed). This time step is of the order of the acoustic time scale which allows to minimize the effect of the time discretization and, thus, allows to remain close to the semi-discrete (time continuous) scheme (the time discretization is not the object of our study although it is also an important question). We compare the results obtained with the Godunov scheme $(\kappa=1)$, the low Mach Godunov scheme $(\kappa=0)$ and the all Mach Godunov scheme $(\kappa=M)$.

In Figure 1, Figure 2 and Figure 3, we plot the norm of $\alpha \mathbf{u}$ in each cell at the initial time and at the final time $t_{\text {final }}=M=10^{-2}$. On a $30 \times 30$ cartesian mesh, the solution given by the Godunov scheme $(\kappa=1)$ is very diffused over time while the solution on a $300 \times 300$ cartesian mesh seems to be close to the initial condition (see Figure 1 and Figure 2). These numerical results illustrate the inaccuracy of the Godunov scheme $(\kappa=1)$ at low Mach number when $M \ll \min (\Delta x, \Delta y$ ) (see Theorem 7.2) and its good behaviour if we use a very fine mesh (i.e. such that $\min (\Delta x, \Delta y)=\mathcal{O}(M)$ : see Point 1 of Theorem 8.1). Moreover, the low Mach Godunov scheme and the all Mach Godunov scheme allow to keep the accuracy at low Mach number even when $M \ll \min (\Delta x, \Delta y)$ (see Points 2 and 3 of Theorem 8.1) since the numerical solutions given by these schemes are near the initial condition (see Figure 1 and Figure 3). 


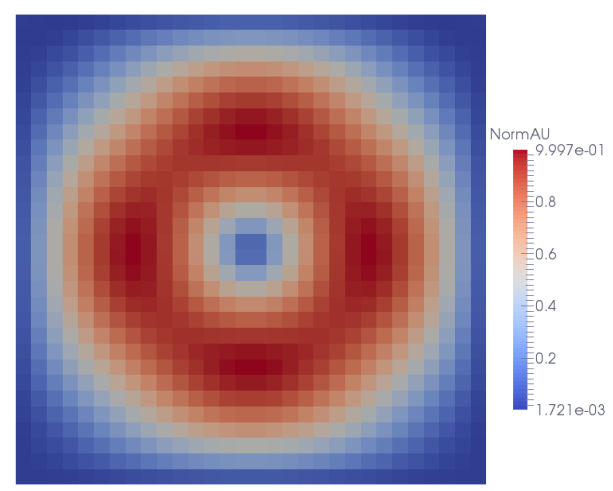

Figure 1. Norm of the velocity $\alpha \mathbf{u}$ at initial time.
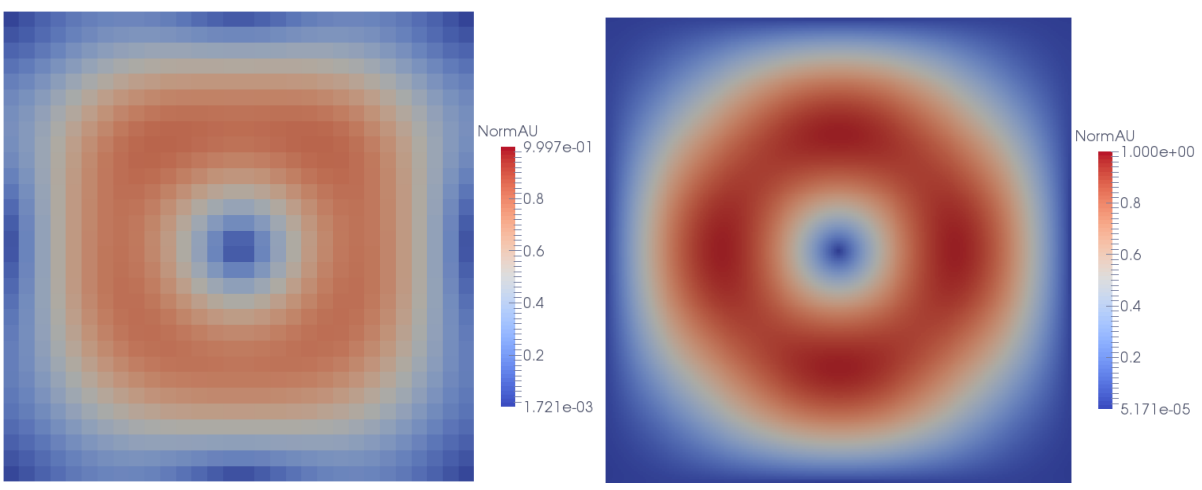

FiguRe 2. Norm of the velocity $\alpha \mathbf{u}$ at final time $t_{\text {final }}=M=10^{-2}$ with the Godunov scheme $(\kappa=1)$ on a $30 \times 30$ cartesian mesh (left picture) and on a $300 \times 300$ cartesian mesh (right picture). On the coarse mesh, the velocity field is very diffused over time while it seems to be close to the initial condition (see Figure 1) for a fine mesh. These numerical results illustrate the inaccuracy of the Godunov scheme $(\kappa=1)$ at low Mach number when $M \ll \min (\Delta x, \Delta y)$ (see Theorem 7.2) and its good behaviour if we use a very fine mesh i.e. such that $\max (\Delta x, \Delta y)=$ $\mathcal{O}(M)$ (see Point 1 of Theorem 8.1).
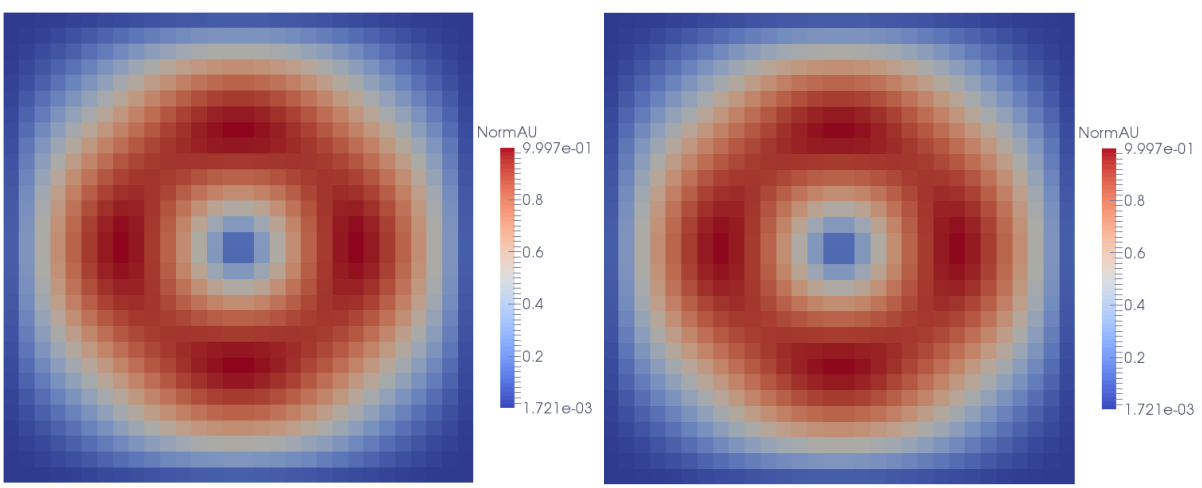

Figure 3. Norm of the velocity $\alpha \mathbf{u}$ at final time $t_{\text {final }}=M=10^{-2}$ with the low Mach Godunov scheme (left picture, $\kappa=0$ ) and the all Mach Godunov scheme (right picture, $\kappa=M$ ) on a $30 \times 30$ cartesian mesh. The low Mach Godunov scheme $(\kappa=0)$ and the all Mach Godunov scheme $(\kappa=M)$ allow to keep the accuracy at low Mach number even when $M \ll \min (\Delta x, \Delta y)$ (see Points 2 and 3 of Theorem 8.1) since the numerical solutions given by these schemes are near the initial condition (see Figure 1). 


\section{Conclusion}

We proposed a low Mach correction and an all Mach correction for the Godunov scheme applied on a cartesian mesh to the linear wave equation with porosity. These corrections have been justified by studying the time behaviour of a solution of the first order modified equations associated to these schemes. It remains to justify these corrections in the discrete cartesian case. The triangular case is also important since we know that the Godunov scheme with a constant porosity is accurate at low Mach number on a triangular (or tetrahedral) mesh $[7,11,16,18]$. These two points are studied in [4]. 


\section{Appendix}

\section{Poincaré-Wirtinger INEQUALity FOR WEIGHTED SPACE}

Proposition. Assume that $\Omega$ is an open convex bounded space in $\mathbb{R}^{d \in\{1,2,3\}}$ and that $\mu$ is a probabilistic measure on $\Omega$ such that $\mu$ and $\frac{1}{\mu}$ are in $L^{\infty}(\Omega)$. Then, we have:

$$
\forall \phi \in H^{1}(\Omega): \quad \int_{\Omega}|\phi(\boldsymbol{x})-\bar{\phi}|^{2} \mu(\boldsymbol{x}) d \boldsymbol{x} \leq 2 \operatorname{diam}(\Omega)^{2}\|\mu\|_{\infty}\left\|\frac{1}{\mu}\right\|_{\infty} \int_{\Omega}|\nabla \phi|^{2} \mu(\boldsymbol{x}) d \boldsymbol{x}
$$

where $\bar{\phi}:=\int_{\Omega} \phi(\boldsymbol{x}) \mu(\boldsymbol{x}) d \boldsymbol{x}$ and $\operatorname{diam}(\Omega):=\sup _{(\boldsymbol{x}, \boldsymbol{y}) \in \Omega^{2}}|\boldsymbol{x}-\boldsymbol{y}|$.

Proof. The proof is done for $d \in\{1,2,3\}$. We have for all $(\mathbf{x}, \mathbf{y}) \in \Omega^{2}$

$$
\begin{array}{r}
\phi(\mathbf{x})-\phi(\mathbf{y})=\int_{0}^{1} \nabla \phi((1-t) \mathbf{x}+t \mathbf{y}) \cdot(\mathbf{x}-\mathbf{y}) d t \Longrightarrow \phi(\mathbf{x})-\bar{\phi}=\int_{\Omega} \int_{0}^{1} \nabla \phi((1-t) \mathbf{x}+t \mathbf{y}) \cdot(\mathbf{x}-\mathbf{y}) d t \mu(\mathbf{y}) d \mathbf{y} \\
\Longrightarrow(\phi(\mathbf{x})-\bar{\phi})^{2} \leq \int_{\Omega} \int_{0}^{1}(\nabla \phi((1-t) \mathbf{x}+t \mathbf{y}) \cdot(\mathbf{x}-\mathbf{y}))^{2} d t \mu(\mathbf{y}) d \mathbf{y} \\
\Longrightarrow(\phi(\mathbf{x})-\bar{\phi})^{2} \leq \int_{\Omega} \int_{0}^{1}|\nabla \phi((1-t) \mathbf{x}+t \mathbf{y})|^{2}|\mathbf{x}-\mathbf{y}|^{2} d t \mu(\mathbf{y}) d \mathbf{y}
\end{array}
$$

with a Jensen inequality (with the function squared) and a Cauchy-Schwartz inequality. By multiplying by $\mu(\mathbf{x})$ and by integrating on $\Omega$, we find

$$
\int_{\Omega}(\phi(\mathbf{x})-\bar{\phi})^{2} \mu(\mathbf{x}) d \mathbf{x} \leq \int_{\Omega} \int_{\Omega} \int_{0}^{1}|\nabla \phi((1-t) \mathbf{x}+t \mathbf{y})|^{2}|\mathbf{x}-\mathbf{y}|^{2} d t \mu(\mathbf{y}) d \mathbf{y} \mu(\mathbf{x}) d \mathbf{x}
$$

which implies that

$$
\int_{\Omega}(\phi(\mathbf{x})-\bar{\phi})^{2} \mu(\mathbf{x}) d \mathbf{x} \leq \operatorname{diam}(\Omega)^{2} \int_{\Omega} \int_{\Omega} \int_{0}^{1}|\nabla \phi((1-t) \mathbf{x}+t \mathbf{y})|^{2} d t \mu(\mathbf{y}) d \mathbf{y} \mu(\mathbf{x}) d \mathbf{x} .
$$

We split the integral by integrating on $\left[0, \frac{1}{2}\right]$ and on $\left[\frac{1}{2}, 1\right]$. This gives

$$
\begin{aligned}
& \int_{\Omega}(\phi(\mathbf{x})-\bar{\phi})^{2} \mu(\mathbf{x}) d \mathbf{x} \leq \operatorname{diam}(\Omega)^{2} \int_{\Omega} \int_{\Omega}\left(\int_{0}^{\frac{1}{2}}|\nabla \phi((1-t) \mathbf{x}+t \mathbf{y})|^{2} d t\right. \\
& \left.+\int_{\frac{1}{2}}^{1}|\nabla \phi((1-t) \mathbf{x}+t \mathbf{y})|^{2} d t\right) \mu(\mathbf{y}) d \mathbf{y} \mu(\mathbf{x}) d \mathbf{x} \\
& \leq \operatorname{diam}(\Omega)^{2}\|\mu\|_{\infty}\left(\int_{\Omega} \int_{\Omega} \int_{0}^{\frac{1}{2}}|\nabla \phi((1-t) \mathbf{x}+t \mathbf{y})|^{2} d t d \mathbf{x} \mu(\mathbf{y}) d \mathbf{y}\right. \\
& \left.+\int_{\Omega} \int_{\Omega} \int_{\frac{1}{2}}^{1}|\nabla \phi((1-t) \mathbf{x}+t \mathbf{y})|^{2} d t d \mathbf{y} \mu(\mathbf{x}) d \mathbf{x}\right) \\
& =\operatorname{diam}(\Omega)^{2}\|\mu\|_{\infty}\left(\int_{\Omega} \int_{0}^{\frac{1}{2}} \int_{\omega(t, \mathbf{y})}|\nabla \phi(\mathbf{z})|^{2} \frac{d \mathbf{z}}{1-t} d t \mu(\mathbf{y}) d \mathbf{y}+\int_{\Omega} \int_{\frac{1}{2}}^{1} \int_{\omega(t, \mathbf{x})}|\nabla \phi(\mathbf{z})|^{2} \frac{d \mathbf{z}}{t} d t \mu(\mathbf{x}) d \mathbf{x}\right)
\end{aligned}
$$


where $\omega(t, \mathbf{x})$ and $\omega(t, \mathbf{y})$ are included in $\Omega$. Thus, by replacing $\omega(t, \mathbf{x})$ and $\omega(t, \mathbf{y})$ with $\Omega$, and since $\frac{1}{1-t} \geq 1$ when $t \in\left[0, \frac{1}{2}\right]$ and $\frac{1}{t} \geq 1$ when $t \in\left[\frac{1}{2}, 1\right]$, we can write

$$
\begin{aligned}
\int_{\Omega}(\phi(\mathbf{x})-\bar{\phi})^{2} \mu(\mathbf{x}) d \mathbf{x} & \leq \operatorname{diam}(\Omega)^{2}\|\mu\|_{\infty} \int_{\Omega}|\nabla \phi(\mathbf{z})|^{2} d \mathbf{z}\left(\int_{\Omega} \int_{0}^{\frac{1}{2}} \frac{d t}{1-t} \mu(\mathbf{y}) d \mathbf{y}+\int_{\Omega} \int_{\frac{1}{2}}^{1} \frac{d t}{t} \mu(\mathbf{x}) d \mathbf{x}\right) \\
& \leq \operatorname{diam}(\Omega)^{2}\|\mu\|_{\infty} \int_{\Omega}|\nabla \phi(\mathbf{z})|^{2} d \mathbf{z}\left(\int_{\Omega} \mu(\mathbf{y}) d \mathbf{y}+\int_{\Omega} \mu(\mathbf{x}) d \mathbf{x}\right)
\end{aligned}
$$

which finally gives

$$
\int_{\Omega}(\phi(\mathbf{x})-\bar{\phi})^{2} \mu(\mathbf{x}) d \mathbf{x} \leq 2 \operatorname{diam}(\Omega)^{2}\|\mu\|_{\infty} \int_{\Omega}|\nabla \phi(\mathbf{z})|^{2} d \mathbf{z}
$$

We obtain (43) by using the previous inequality and by noting that for all $\mathbf{z} \in \Omega$, we have $1 \leq\left\|\frac{1}{\mu}\right\|_{\infty} \mu(\mathbf{z})$.

\section{REFERENCES}

[1] M. Bernard, S. Dellacherie, G. Faccanoni, B. Grec, and Y. Penel. Study of a low Mach nuclear core model for two-phase flows with phase transition I: stiffened gaz law. Math. Mod. and Num. Anal., 48(6):1639-1679, 2014.

[2] S. Dellacherie. Analysis of Godunov type schemes applied to the compressible Euler system at low Mach number. J. Comp. Phys., 4(229):978-1016, 2010.

[3] S. Dellacherie, G. Faccanoni, B. Grec, E. Nayir, and Y. Penel. 2D numerical simulation of a low Mach nuclear core model with stiffened gas using FreeFem++. ESAIM:ProcS, 45:138-147, 2014.

[4] S. Dellacherie, J. Jung, and P. Omnes. An all Mach correction for the Godunov scheme applied to the linear wave equation with porosity. In preparation, 2015.

[5] S. Dellacherie, J. Jung, P. Omnes, and P.-A. Raviart. Construction of modified Godunov type schemes accurate at any Mach number for the compressible Euler system. Preprint HAL 2015, https://hal.archives-ouvertes.fr/hal-00776629.

[6] S. Dellacherie and P. Omnes. On the Godunov scheme applied to the variable cross-section linear equation. Finite Volumes for Complex Applications VI (FVCA6). Problems and Perspectives, 4:313-321, 2011.

[7] S. Dellacherie, P. Omnes, and F. Rieper. The influence of cell geometry on the Godunov scheme applied to the linear wave equation. J. Comp. Phys., 229(14):5315-5338, 2010.

[8] F. Fillion, A. Chanoine, S. Dellacherie, and A. Kumbaro. FLICA-OVAP: A new platform for core thermal-hydraulic studies. Nuclear Engineering and Design, 241(11):4348-4358, 2011.

[9] E. Godlewski and P.-A. Raviart. Hyperbolic systems of conservation laws. Société de Mathématiques Appliquées et Industrielles Marketing, 1991.

[10] E. Godlewski and P.-A. Raviart. Numerical approximation of hyperbolic systems of conservation laws. Applied Mathematical Sciences 118. Springer-Verlag, New York, 1996.

[11] H. Guillard. On the behavior of upwind schemes in the low Mach number limit. IV: P0 approximation on triangular and tetrahedral cells. Computers \& Fluids, 38(10):1969-1972, 2009.

[12] H. Guillard and A. Murrone. On the behavior of upwind schemes in the low Mach number limit: II. Godunov type schemes. Computers 85 Fluids, 33(4):655-675, 2004.

[13] H. Guillard and A. Murrone. Behavior of upwind scheme in the low Mach number limit: III. Preconditioned dissipation for a five equation two phase model. Computers \& Fluids, 37(10):1209-1224, 2008.

[14] H. Guillard and C. Viozat. On the behaviour of upwind schemes in the low Mach number limit. Computers 8 Fluids, 28(1):6386, 1999.

[15] Y. Moguen, S. Dellacherie, P. Bruel, and E. Dick. Momentum interpolation for quasi one-dimensional unsteady low Mach number flows with acoustics. 11 th World Congress on Computational Mechanics (WCCM XI), ECCOMAS, 2014.

[16] F. Rieper. On the dissipation mechanism of upwind-schemes in the low Mach number regime: A comparison between Roe and HLL. J. Comp. Phys., 229(2):221-232, 2010.

[17] F. Rieper. A low Mach number fix for Roe's approximate Riemann solver. J. Comp. Phys., 230(13):5263-5287, 2011.

[18] F. Rieper and G. Bader. The influence of cell geometry on the accuracy of upwind schemes in the low Mach number regime. J. Comp. Phys., 228(8):2918-2934, 2009. 